\title{
Pulling the boundary into the bulk
}

\author{
Yasunori Nomura, ${ }^{1,2,3}$ Pratik Rath, ${ }^{1,2}$ and Nico Salzetta ${ }^{1,2}$ \\ ${ }^{1}$ Berkeley Center for Theoretical Physics, Department of Physics, University of California, \\ Berkeley, CA 94720, USA \\ ${ }^{2}$ Theoretical Physics Group, Lawrence Berkeley National Laboratory, Berkeley, California 94720, USA \\ ${ }^{3}$ Kavli Institute for the Physics and Mathematics of the Universe (WPI), \\ The University of Tokyo Institutes for Advanced Study, Kashiwa 277-8583, Japan
}

(Received 9 May 2018; published 6 July 2018)

\begin{abstract}
Motivated by the ability to consistently apply the Ryu-Takayanagi prescription for general convex surfaces and the relationship between entanglement and geometry in tensor networks, we introduce a novel, covariant bulk object - the holographic slice. The holographic slice is found by considering the continual removal of short-range information in a boundary state. It thus provides a natural interpretation as the bulk dual of a series of coarse-grained holographic states. The slice possesses many desirable properties that provide consistency checks for its boundary interpretation. These include the monotonicity of both area and entanglement entropy, uniqueness, and the inability to probe beyond late-time black hole horizons. Additionally, the holographic slice illuminates physics behind entanglement shadows, as minimal-area extremal surfaces anchored to a coarse-grained boundary may probe entanglement shadows. This lets the slice flow through shadows. To aid in developing intuition for these slices, many explicit examples of holographic slices are investigated. Finally, the relationship to tensor networks and renormalization (particularly in AdS/CFT) is discussed.
\end{abstract}

DOI: $10.1103 /$ PhysRevD.98.026010

\section{INTRODUCTION}

The holographic duality between asymptotically anti-de Sitter (AdS) spacetimes in $d+1$ dimensions and conformal field theories (CFTs) in $d$ dimensions is perhaps the closest realization of a complete theory of quantum gravity [1-3]. One of the most intriguing results stemming from this correspondence is the renowned Ryu-Takayanagi formula relating entanglement entropy in time-independent CFTs to the area of minimal surfaces in the bulk spacetime [4]. The covariant extension of this formula to include time-dependent cases was obtained by Hubeny, Rangamani, and Takayanagi and uses extremal bulk surfaces (henceforth referred to as the HRRT prescription) [5]. Remarkably, by including quantum corrections to this formula, one obtains entanglement wedge reconstruction [6,7]. These investigations have shed light on the deep connection between entanglement in the boundary and emergent gravitational physics in the bulk.

However, there is reason to believe that these results extend beyond the scope of AdS/CFT. The BekensteinHawking formula [8,9] and the covariant entropy bound [10] provide us with holographic bounds on entropy in

Published by the American Physical Society under the terms of the Creative Commons Attribution 4.0 International license. Further distribution of this work must maintain attribution to the author(s) and the published article's title, journal citation, and DOI. Funded by SCOAP . general spacetimes. These suggest that gravitational physics may inherently be holographic [11,12]. Furthermore, the areas of extremal surfaces anchored to any convex boundaries satisfy all known entropic inequalities [13-16]. In isometric tensor networks, calculating the entanglement entropy of a subregion of boundary sites reduces to finding the minimum cut across the network [17]. A version of entanglement wedge reconstruction also holds in perfect and random tensor networks $[18,19]$. This evidence seems to indicate that the HRRT prescription may in fact generalize to spacetimes outside of AdS.

It is with this perspective that we have pursued investigations of quantum gravity beyond AdS/CFT. We postulate that the HRRT prescription (with quantum corrections $[20,21]$ to allow for entanglement wedge reconstruction) applies to general convex boundaries. In particular, we assume the existence of a quantum state that "lives" on the convex boundary and encodes the bulk geometry of the interior. Our previous work [22-24] has focused primarily on investigating this assumption applied to holographic screens [25], but holographic screens are only special in the sense that they are the largest surfaces in which we can apply the HRRT prescription in general spacetimes. In this paper, we relax this condition and look at general convex surfaces; in particular this allows us to consider asymptotically AdS spacetimes.

We emphasize that the postulate we adopt here is falsifiable. At any point in the analysis, had the geometric 
properties of general relativity prohibited a consistent boundary interpretation, the program would have failed. Through the present, however, no such roadblock has presented itself. In fact, we can view the self-consistency of this work as further evidence that the relationship between entanglement and geometry prevails in general spacetimes.

The present framework allows us to consider a nested family of convex surfaces each of which contains less bulk information than the previous one. Taking a natural continuum version of these convex surfaces yields a surface which we dub the holographic slice. As has proved historically useful, studying covariantly defined geometric objects yields insights into holographic theories, and the holographic slice is such an object. In particular, the slice allows us to visualize the coarse graining of holographic states and provides us with a method to categorize bulk regions as being "more IR" than others.

The construction of the slice is purely perturbative, and thus does not allow us to analyze complex quantum gravitational states formed as superpositions of many geometries. It is inherently tied to one background geometry, but this is no more restrictive than the HRRT prescription itself. For a simply connected boundary, the slice seems to sweep the maximal bulk region that can be perturbatively reconstructed, i.e., it pulls the boundary in through shadows all the way to black hole horizons, and contracts no further. It was the study of extremal surfaces in maximally entangled pure states that initiated this work, where it was realized that maximally entangled states were fixed points of flow in the direction of small HRRT surfaces [24]. ${ }^{1}$

\section{A. Overview}

In Sec. II, we offer the intuitive motivation for the construction of the holographic slice. This reveals the connection to coarse graining and provides a basic definition for the object. In Sec. III, we geometrically define the object rigorously and then illustrate some of its most important properties. These properties must necessarily be satisfied for a consistent interpretation as coarse graining of a boundary state. Section IV goes through multiple explicit examples of the slice in different spacetimes.

After introducing the holographic slice as a geometric object, we are poised to analyze its boundary interpretation in Sec. V. Here we delve into its relationship to coarse graining and emphasize that the slice encodes a sequence of codimension- 0 bulk regions, not merely the codimension- 2 bulk convex surfaces. We also describe the relationship to tensor networks, particularly continuous tensor networks [27]. Because the holographic slice is constructed from one boundary time slice, it can be used as a novel gauge fixing

\footnotetext{
${ }^{1}$ By maximally entangled, we mean that the von Neumann entropy of any subregion saturates the Page curve [26]. This was referred to as maximally entropic in Ref. [24].
}

of the bulk; this is discussed in the final subsection of Sec. V. Since the slice grants us a way to uniquely pull in the boundary, it is natural to consider its connection to renormalization. This is explored in Sec. VI. We conclude with discussing the slice's place in the wider view of quantum gravity in Sec. VII. The appendices contain proofs of various geometric statements made in the body of the text.

\section{B. Preliminaries}

This paper will work in the framework of holography for general spacetimes proposed in Ref. [22]. We will highlight the applications to AdS/CFT but use language from generalized holography. In particular, the term "boundary" will refer to the holographic screen, which reduces to the conformal boundary of AdS. Additionally, holographic screens have a unique time foliation into codimension-2 surfaces called leaves [28]. This uniqueness is lost in the case of AdS because of the asymptotic symmetries, but to remain consistent within the generalized framework we must choose a particular time foliation of the boundary of AdS [23]. We will then refer to a time slice of this foliation as a leaf.

For a subregion, $A$, of a leaf, we will denote its HRRT surface as $\gamma(A)$ and its entanglement wedge as $\operatorname{EW}(A)$. $\operatorname{EW}(A)$ is defined as the domain of dependence of any closed, compact, achronal set with boundary $A \cup \gamma(A){ }^{2}$ Throughout this paper, we will work at the lowest order in bulk Newton's constant. In particular, we will only consider extremal surfaces found by extremizing the area, not the generalized entropy. We expect that by making appropriate modifications, along the lines of Refs. [20,21,29], our results can be extended to higher orders.

For an achronal codimension-2 surface $\omega$, we denote the domain of dependence of any achronal set with boundary $\omega$ as $D(\omega)$. Wherever $G_{\mathrm{N}}=l_{\mathrm{P}}^{d-1}$ appears, it represents Newton's constant in the bulk spacetime.

\section{MOTIVATION}

Amongst other things, the concept of subregion duality in holographic theories allows us to address questions regarding where bulk information is stored in the boundary theory [6,7,30-36]. This line of inquiry has provided us with the intuition that bulk geometric information is encoded redundantly in the boundary theory. In particular, a bulk local operator can be represented in multiple

\footnotetext{
${ }^{2}$ In standard AdS/CFT, reflective boundary conditions are imposed at the conformal boundary. This extends the domain of dependence for $A \cup \gamma(A)$ to include the boundary domain of dependence of $A$. However, for holographic screens there are no such impositions on the screen (they can even be spacelike), and thus $\operatorname{EW}(A)$ generally does not include any portion of the screen other than $A$ itself. In particular, there is no generalization of causal wedges to holographic screens.
} 
different regions of the boundary, a special case being the whole boundary space. However, despite this seemingly democratic distribution of bulk information throughout boundary degrees of freedom (d.o.f.), lack of access to a boundary region necessarily prohibits the reconstruction of a corresponding bulk region. Namely, if one removes a subregion $A$ from a leaf $\sigma$, the maximum possible bulk region reconstructed from the remaining region, $\bar{A}$, will be the entanglement wedge of $\bar{A}, \operatorname{EW}(\bar{A})$. This implies that indispensable information of the region $\operatorname{EW}(A)$ is stored in $A$.

Suppose one were to coarse grain over all boundary subregions of balls of radius $\delta$. From the logic above, the bulk region whose information can remain is given by

$$
R\left(B_{\delta}\right)=\bigcap_{p \in \sigma} \operatorname{EW}\left(\overline{B_{\delta}(p)}\right),
$$

where $B_{\delta}(p)$ is a ball of radius $\delta$ centered at point $p$ on the boundary leaf $\sigma$. Because the intersection of domains of dependence is itself a domain of dependence, ${ }^{3} R\left(B_{\delta}\right)$ is a domain of dependence of some achronal set, and thus has a unique boundary, $\sigma\left(B_{\delta}\right)$.

Motivated by ideas of holographic renormalization in AdS/CFT [37-41], surface/state correspondence [13], and previous work on holographic screens [15,22-24], we conjecture that there exists a holographic state living on $\sigma\left(B_{\delta}\right)$ which encodes aspects of the bulk geometry to its interior. A check of this proposal is that the HRRT prescription can be consistently applied, in the sense that the areas of these HRRT surfaces obey the known holographic entropy inequalities. Given this consistency check, we conjecture that "coarse-grained subregion duality" holds-namely that entanglement wedge reconstruction holds on this new leaf.

Now suppose one wants to coarse grain over some scale on this new leaf, $\sigma\left(B_{\delta}\right)$. This will produce a new leaf even deeper in the bulk. Repeating this process will in turn produce a series of new leaves, henceforth called renormalized leaves. Sending the coarse-graining scale at each step to zero in a consistent manner will produce a continuous family of renormalized leaves that sweep out a smooth surface through the bulk, $\Upsilon$. The manner in which $\Upsilon$ is constructed naturally reveals its relationship to holographic coarse graining. This prompts us to assert that the continuous coarse graining of a holographic state pulls the boundary slice in along the slice $\Upsilon$.

\section{HOLOGRAPHIC SLICE}

The geometric object $\Upsilon$ is what we will refer to as the holographic slice. In this section, we give a more rigorous definition of holographic slices and highlight some of the salient properties of them.

\footnotetext{
${ }^{3}$ We could not find a proof of this statement, so we have included one in Appendix A.
}

\section{A. Definition}

Consider a closed codimension-2 achronal surface $\sigma$ living in a $(d+1)$-dimensional spacetime $M$. Denote the two future-directed null orthogonal directions as $k$ and $l$. Suppose the null expansions along these directions satisfy $\theta_{k} \leq 0$ and $\theta_{l}>0 .{ }^{4}$ For concreteness, one could imagine $\sigma$ to be a leaf of a past holographic screen $\left(\theta_{k}=0, \theta_{l}>0\right)$ or a time slice of the (regularized) boundary of AdS. Borrowing this language, we will call $\sigma$ a leaf. From Ref. [15], we know that the boundary of the domain of dependence of $\sigma, D(\sigma)$, is an extremal surface barrier for HRRT surfaces anchored to $\sigma$. In addition, the boundary of an entanglement wedge of a subregion $\Gamma$ on $\sigma$ serves as an extremal surface barrier for all extremal surfaces anchored within $\mathrm{EW}(\Gamma)$.

Now, on $\sigma$, consider a family of open, codimension- 0 (within the leaf) smooth subregions, with an injective mapping from points on the leaf, $p$, to subregions, $C(p)$, with the constraint that $p \in C(p)$ and that $C(p)$ varies continuously with $p$. For example, one may take $C(p)$ to be open balls of radius $\delta$ centered at $p, B_{\delta}(p)$. Now, let

$$
R(C)=\bigcap_{p \in \sigma} \operatorname{EW}(\overline{C(p)}),
$$

where $\overline{C(p)}$ is the complement of $C(p)$ in $\sigma$. From Appendix A, we know that $R(C)$ itself is a domain of dependence of some achronal sets, all of which share a unique boundary, $\sigma_{C}^{1}$, called a renormalized leaf; see Fig. 1.

Provided the characteristic scale of $\operatorname{EW}(C(p))$ is sufficiently smaller than the extrinsic curvature scale of $\sigma$, we can identify all points on $\sigma_{C}^{1}$ with those on $\sigma$. At each point on $\sigma$, consider the plane generated by $k$ and $l$. This will intersect $\sigma_{C}^{1}$ at one point so long as adjacent planes do not intersect at a caustic before hitting $\sigma_{C}^{1}$, which is guaranteed if we take $C(p)$ to be sufficiently small. We now have a natural identification of the points on $\sigma$ with points on $\sigma_{C}^{1}$. In particular, we can identify the length scale $\delta$ on $\sigma$ to the appropriate scale on $\sigma_{C}^{1}$. Moreover, since $\sigma_{C}^{1} \subseteq$ $\operatorname{EW}(\overline{C(p)}) \forall p$ and the boundary of $\operatorname{EW}(\overline{C(p)})$ serves as an extremal surface barrier for all surfaces anchored within $\operatorname{EW}(\overline{C(p)})$, all extremal surfaces anchored to $\sigma_{C}^{1}$ are contained within $R(C)=D\left(\sigma_{C}^{1}\right)$. If we interpret the area of these extremal surfaces as entanglement entropies for the associated subregions, the convexity of $R(C)$ and the null energy condition ensure that all known holographic entanglement inequalities are satisfied. This allows us to interpret these extremal surfaces as HRRT surfaces.

Utilizing coarse-grained subregion duality, we can repeat the construction above but with new subregions, $C^{1}(p)$, on the renormalized leaf, $\sigma_{C}^{1}$. This yields a new leaf $\sigma_{C}^{2}$. We can

\footnotetext{
${ }^{4}$ Appropriate modifications can be made to extend to surfaces where $\theta_{k} \geq 0$ and $\theta_{l}<0$.
} 


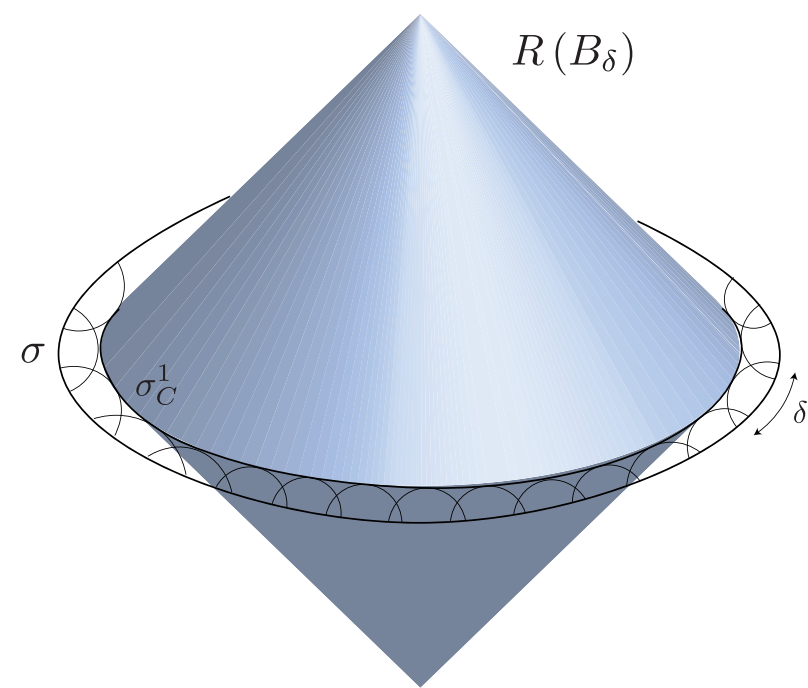

FIG. 1. $R\left(B_{\delta}\right)$ is the entanglement wedge associated with the new leaf $\sigma_{C}^{1}$, where we have taken $C(p)=B_{\delta}(p)$. It is formed by intersecting the entanglement wedges associated with the complements of spherical subregions of size $\delta$ on the original leaf $\sigma$.

associate points on $\sigma_{C}^{2}$ with those on $\sigma_{C}^{1}$, and hence on $\sigma$, as was done above. All we require of the new subregions is that the size scale of $C^{i}(p)$ match with that of $C(p)$ under the natural identification described previously. This procedure can be repeated until stringy effects become important, i.e., when the size of the renormalized leaf is $O\left(l_{\mathrm{s}}\right)$.

In the limit of sending the size of $C(p)$ to 0 for all $p$, the collection of all renormalized leaves in $M, \Upsilon=\left\{\sigma_{C}^{i}\right\}$ sweeps out a continuous surface. This is a holographic slice. Note that we can take the $G_{\mathrm{N}} \rightarrow 0$ limit in discussing classical spacetime, so we may take $C(p) \rightarrow 0$ thus making $\Upsilon$ continuous in this limit. We can then label the renormalized leaves of $\Upsilon$ by some continuous parameter $\lambda$, corresponding to the depth of the renormalized leaf, i.e., $\sigma(\lambda)$ is some $\sigma_{C}^{i}$ and $\sigma(0)=\sigma$. Below we take $\lambda$ to decrease as $i$ increases, so that $\lambda \leq 0$.

\section{B. Properties}

\section{Uniqueness}

$\Upsilon$ is dependent on an extraordinary number of d.o.f., namely the shape $C(p)$ at each point on $\sigma$. Despite this freedom, we find that $\Upsilon$ is unique provided some mild assumptions hold.

In particular, imposing homogeneity and isotropy on $C(p)$ (and each subsequent $C^{i}(p)$ ) yields a unique holographic slice. For example, one can restrict themselves to the case where $C^{i}(p)$ are composed of the same shape and with random orientations. These all reduce to the slice formed by considering balls of constant radius for $C(p)$ and mapping these balls to the subsequent renormalized leaves. We will focus on this preferred slice for the remainder of the paper.
A full discussion of uniqueness is provided in Appendix B. However, one of the primary results is that the vector $s$, which is tangent to $\Upsilon$ and radially evolves the leaf inward is given by

$$
s=\frac{1}{2}\left(\theta_{k} l+\theta_{l} k\right) .
$$

This tells us that for a (nonrenormalized) leaf of a holographic screen, $s \propto k$ and $\theta_{s}=0$. In these situations, the holographic slice initially extends in the null direction and the leaf area remains constant.

In fact, the $s$ vector coincides with the Lorentzian generalization of the mean curvature vector of the leaf $[42,43]$. This preferred holographic slice is then realized as the mean curvature flow of the initial leaf.

\section{Monotonicity of renormalized leaf area}

One of the most important features of the holographic slice is that the area of the renormalized leaves decreases monotonically as $\lambda$ decreases. This is crucial to the interpretation as coarse graining. This property can be shown in a manner similar to Ref. [44], but only after showing that $\theta_{k} \leq 0$ and $\theta_{l} \geq 0$ for each renormalized leaf. This is proved in Appendix C.

Armed with this knowledge, consider a point $p$ on $\sigma$ and the $s$ vector (defined in the previous section) orthogonal to the leaf $\sigma$ at $p$. The integral curves of $s$ passing through $p$ provide a mapping of $p$ to a unique point on each $\sigma(\lambda)$. Now consider an infinitesimal area element $\delta A$ around $p$. The rate at which this area changes as one flows along $s$ is measured by the expansion

$$
\theta_{s}=\theta_{k} \theta_{l} \leq 0,
$$

as found in Appendix B.

Since the area for all infinitesimal area elements decreases on flowing along $s$, the total leaf area also decreases. In fact, this property holds locally. For any subregion of a renormalized leaf, as one flows inward along the holographic slice the area of the subregion decreases monotonically.

\section{Monotonicity of entanglement entropy}

Along with the fact that the renormalized leaf area shrinks, the entanglement entropy of subregions also decreases monotonically. This must necessarily happen for a consistent interpretation that the coarse-graining procedure continuously removes short-range entanglement. This is precisely the spacelike monotonicity theorem of Ref. [22], so we will only sketch the idea. ${ }^{5}$

Consider a leaf $\sigma=\sigma\left(\lambda_{0}\right)$ and a renormalized leaf obtained after some small amount of radial evolution,

\footnotetext{
${ }^{5}$ This interpretation may in fact be the most natural explanation of why the spacelike monotonicity theorem holds.
} 
$\sigma^{\prime}=\sigma\left(\lambda_{0}+\delta \lambda\right)$, where $\delta \lambda<0$. A subregion $A$ of $\sigma$ is mapped to subregion $A^{\prime}$ of $\sigma^{\prime}$ by following the integral curves of $s$.

Suppose the HRRT surface $\gamma\left(A^{\prime}\right)$ anchored to $A^{\prime}$ is the minimal surface on a spacelike slice $\Sigma^{\prime}$. Now extend $\Sigma^{\prime}$ by including the portion of the holographic slice between $\sigma$ and $\sigma^{\prime}$, such that $\Sigma^{\prime}$ is now an achronal slice with boundary $\sigma$. Now consider the minimal surface $\Xi(A)$ anchored to $A$ on this extended $\Sigma^{\prime} . \Xi(A)$ has a portion in the exterior of $\sigma^{\prime}$ which can be projected down to $\sigma^{\prime}$ using the normal vector $s$. This projection, denoted by $\Xi(A) \rightarrow \pi(\Xi(A))$, decreases the area of $\Xi(A)$ due to the spacelike signature of $\Sigma^{\prime}$. This projection results in a surface anchored to $A^{\prime}$, which must have an area greater than that of $\gamma\left(A^{\prime}\right)$. On the other hand, due to the maximin procedure [35], $\Xi(A)$ must also have an area less than the area of the HRRT surface $\gamma(A)$ anchored to $A$. In summary,

$$
\left\|\gamma\left(A^{\prime}\right)\right\| \leq\|\pi(\Xi(A))\| \leq\|\Xi(A)\| \leq\|\gamma(A)\|
$$

where $\|x\|$ represents the volume of the object $x$ (often called the area for a codimension-2 surface in spacetime). The inequalities arise from minimization, projection, and maximization, respectively.

\section{Subregion flow contained within entanglement wedge}

Suppose one were given access to a finite subregion $A$ on the leaf $\sigma$ and chose to apply the holographic slice construction only to this subregion. The result would be a sequence of renormalized leaves given by $\sigma(\lambda)=A(\lambda) \cup \bar{A}$, with $A(\lambda)$ denoting the sequence of subregions that result from radially evolving $A$ as illustrated in Fig. 2.

An interpretation of this procedure as coarse graining requires that it should not add any further information than what was already available. Thus, it should not allow one to reconstruct points in the bulk beyond what was already accessible from $A$, i.e., $\mathrm{EW}(A)$. This is ensured by the fact that the boundary of $\operatorname{EW}(A)$ acts as an extremal surface barrier for HRRT surfaces anchored to points inside $\operatorname{EW}(A)$, and thus, at no step does $A(\lambda)$ cross outside $\operatorname{EW}(A)$. In fact, if there were a nonminimal extremal surface anchored to $A$ which is contained within $\operatorname{EW}(A)$, the holographic slice would not be able to go beyond this. This would be the case if one were to consider $A$ to be a large subregion of the boundary dual to an AdS black hole.

\section{Probes the directly reconstructable region}

Using the definition of Ref. [23], we define the directly reconstructable region of spacetime as the set of bulk points which can be localized by the intersections of some set of boundary anchored HRRT surfaces and the boundary of their entanglement wedges. Boundary operators corresponding to the maximally localizable bulk operators are dual to local operators in the directly reconstructable region [45].

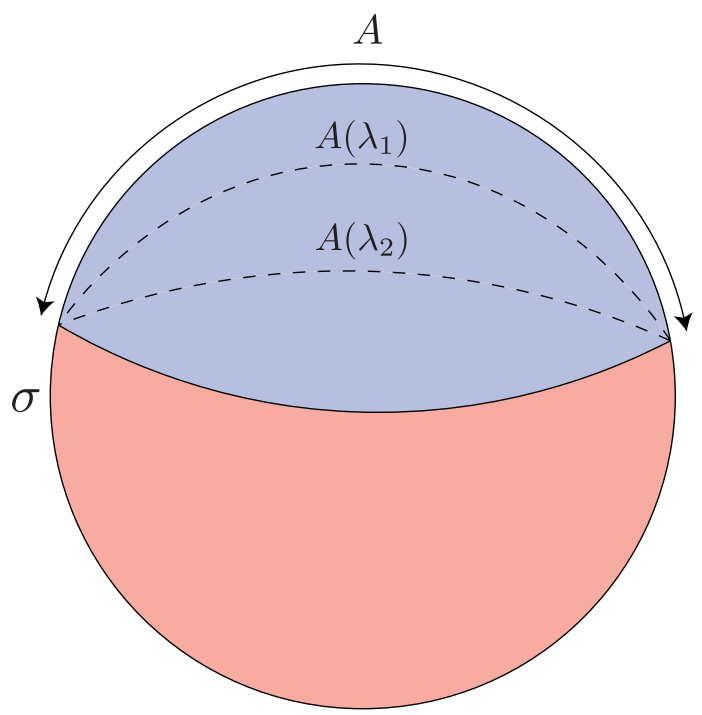

FIG. 2. The radial evolution procedure when restricted to a subregion $A$ results in a new leaf $\sigma(\lambda)=A(\lambda) \cup \bar{A}$, where $A$ is mapped to a subregion $A(\lambda)$ contained within $\operatorname{EW}(A)$ (blue). The figure illustrates this for two values of $\lambda$ with $\lambda_{2}<\lambda_{1}<0$ (dashed lines).

As argued in Ref. [23], the interior of an equilibrated black hole cannot be reconstructed using the intersection of entanglement wedges. Since the horizon acts as a barrier for all extremal surfaces anchored to points outside the black hole, $\sigma(\lambda)$ stays outside the horizon at each step. Thus, the holographic slice cannot enter the black hole interior. This implies that bulk regions that are not directly reconstructable using the entire holographic screen are inaccessible to any holographic slice.

In fact, as long as $\sigma(\lambda)$ does not become extremal, the holographic slice procedure can continue moving the leaf spatially inward. This is a consequence of Theorem 1 in Ref. [24]. As we will see later, the radial evolution will only halt once the boundary state on the renormalized leaf no longer has distillable local correlations. This can happen in two ways. The first is that the surface closes off to zero area (corresponding to the vanishing of the coarsegrained Hilbert space). The second is if the surface asymptotes to a bifurcation surface or Killing horizon (corresponding to a maximally entangled state).

Holographic slices probe entanglement shadows, the spacetime regions which cannot be probed by HRRT surfaces anchored to a nonrenormalized leaf $\sigma$. The extremal surfaces anchored to $\sigma$ that probe the shadow regions are nonminimal. This prevents the reconstruction of points in these regions by using the intersection of HRRT surfaces anchored to $\sigma$. However, the set of HRRT surfaces used for constructing subsequent renormalized leaves need not be minimal on $\sigma$; they need only be minimal on the renormalized leaf at hand. Since nonminimal surfaces anchored to $\sigma$ become minimal when anchored to an appropriately renormalized leaf $\sigma(\lambda)$, the holographic slice 
can flow through entanglement shadows. We will see an example of this in the next section.

Not only will the holographic slice itself flow through entanglement shadows, HRRT surfaces anchored to a renormalized leaf will probe regions behind shadows of the original leaf. Again, this is because the minimal extremal surfaces anchored to renormalized leaves need not be portions of minimal extremal surface anchored to the original leaf. In fact, one immediately starts recovering portions of shadow regions once the boundary is pulled in. This is what occurs in dense stars and conical AdS. In these cases, more and more of the shadow is recovered as the boundary is pulled in, and the entire shadow is only recovered once the slice contracts to a point. ${ }^{6}$

Because the holographic slices pass through shadows, it seems that the collection of all holographic slices anchored to boundary leaves will sweep out the directly reconstructable region. However, this breaks down if one considers a disconnected boundary. Consider the case of a two-sided AdS black hole. The directly reconstructable region can include regions behind the horizon if one picks a foliation of the left and right boundaries with an offset in time (see Appendix A of Ref. [24]). The intersection of HRRT surfaces anchored to large subregions with support on both boundaries allow for the reconstruction of points behind the horizon. On the other hand, the holographic slice is built from infinitesimal HRRT surfaces anchored to individual boundaries and hence cannot recover regions built from these long-range correlations. In the two-sided black hole (no matter what the offset in boundary times), the holographic slice will always connect through the bifurcation surface and never probe behind the horizon; Sec. IV B explains this in detail.

\section{EXAMPLES}

In this section, we illustrate salient properties of the holographic slice using a few example spacetimes.

\section{A. Conical AdS}

We first consider conical $\mathrm{AdS}_{3}$ to illustrate that the holographic slice probes regions inside entanglement shadows. In order to obtain conical $\mathrm{AdS}_{3}$, we start with the $\mathrm{AdS}_{3}$ metric

$d s^{2}=-\left(1+\frac{r^{2}}{L^{2}}\right) d t^{2}+\left(1+\frac{r^{2}}{L^{2}}\right)^{-1} d r^{2}+r^{2} d \theta^{2}$

where $L$ is the AdS length scale. We then perform a $\mathbb{Z}_{n}$ quotient, so that the angular coordinate $\theta$ has periodicity

\footnotetext{
${ }^{6}$ This will be explored in future work. This is similar to how entanglement of purification probes portions of shadow regions [46]. However, to recover the entire shadow region using entanglement of purification, one must impose additional conditions on the purification $[47,48]$.
}

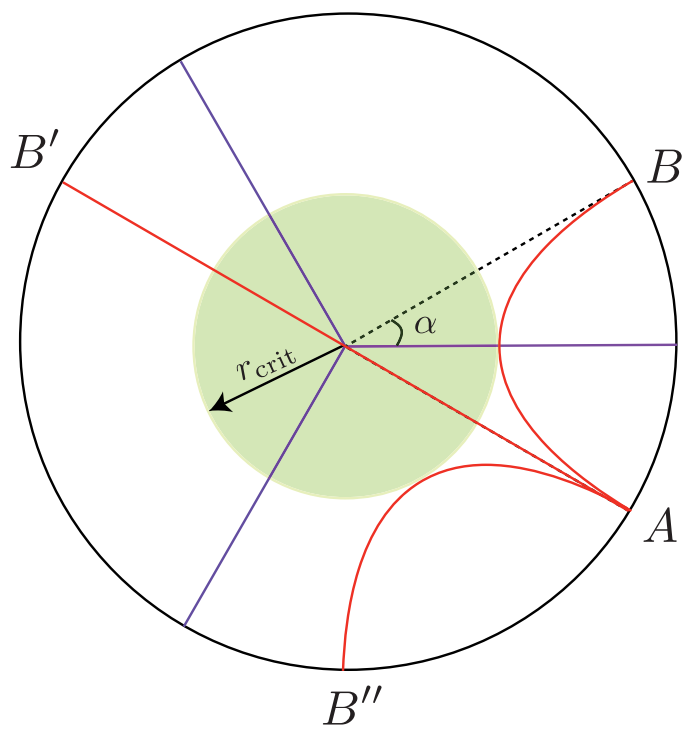

FIG. 3. The case of conical $\mathrm{AdS}_{3}$ with $n=3$. The points $B, B^{\prime}$, and $B^{\prime \prime}$ are identified. There are three geodesics from $A$ to $B$, of which generically only one is minimal. Here, we have illustrated the subregion $A B$ with $\alpha=\pi / 6$, where two of the geodesics are degenerate. This is the case in which the HRRT surface probes deepest into the bulk, leaving a shadow region in the center. Nevertheless, the holographic slice spans the entire spatial slice depicted.

$2 \pi / n$. Locally, this spacetime is identical to $\mathrm{AdS}_{3}$, and solves the Einstein equations for a negative cosmological constant away from $r=0$. However, there is a conical defect at $r=0$ introduced by the $\mathbb{Z}_{n}$ quotient.

HRRT surfaces in this spacetime simply correspond to minimal-length geodesics anchored to subregions at the conformal boundary. As illustrated in Fig. 3, there are $n$ geodesics in the parent $\mathrm{AdS}_{3}$ spacetime which are candidate extremal surfaces for a given subregion. However, generically only one of them is minimal and corresponds to the HRRT surface. The geodesics in $\mathrm{AdS}_{3}$ are described by the equation

$$
\tan ^{2} \theta=\frac{r^{2} \tan ^{2} \alpha-L^{2}}{r^{2}+L^{2}}
$$

where $\alpha$ is the half-opening angle of the subregion being considered. Since the angular coordinate $\theta$ has a periodicity of $2 \pi / n$, the minimal-length geodesic that probes deepest into the bulk is obtained when $\alpha=\pi / 2 n$. From Eq. (7), this gives a critical radius of [49]

$$
r_{\text {crit }}(n)=L \cot \left(\frac{\pi}{2 n}\right)
$$

which takes a nonzero finite value for $n \neq 1$. Thus, the region $r<r_{\text {crit }}(n)$ is an entanglement shadow, which cannot be probed by HRRT surfaces anchored to the conformal boundary. 
The holographic slice is constructed by finding infinitesimal HRRT surfaces starting from the (regularized) conformal boundary. Since the spacetime is locally $\mathrm{AdS}_{3}$, the HRRT surfaces for small regions are identical to those in $\mathrm{AdS}_{3}$. Because of the static and spherically symmetric nature of $\mathrm{AdS}_{3}$, the renormalized leaves correspond to surfaces of constant $r$ and $t$. Now, since $r_{\text {crit }}(n)$ is not an extremal surface barrier, as can be seen from the existence of nonminimal extremal surfaces penetrating it, the holographic slice suffers no obstruction in crossing over to the entanglement shadow. This implies that the holographic slice is simply given by a constant time slice that covers all of the spatial region $r \in[0, \infty)$.

In general, holographic slices do not have any difficulty in going into entanglement shadow regions, since these shadows are not associated with extremal surface barriers which any extremal surfaces anchored to the outside cannot penetrate [50]. In fact, holographic slices also sweep entanglement shadows other than those in the centers of conical AdS, e.g., regions around a dense star [51].

\section{B. Black holes}

Consider a two-sided eternal $\mathrm{AdS}_{d+1}$ Schwarzschild black hole. The metric is given by

$$
d s^{2}=-f(r) d t^{2}+\frac{1}{f(r)} d r^{2}+r^{2} d \Omega_{d-1}^{2}
$$

where

$$
f(r)=1+\frac{r^{2}}{L^{2}}-\left(\frac{r_{+}}{r}\right)^{d-2}\left(1+\frac{r_{+}^{2}}{L^{2}}\right),
$$

with $r_{+}$being the horizon radius. As can be seen in Fig. 4, the two exterior regions have a timelike Killing vector. Thus, the HRRT surfaces anchored to subregions with support only on one boundary respect the Killing symmetry and lie on the constant $t$ slice connecting the respective boundary to the bifurcation surface. Subregions anchored on both boundaries could potentially lie on a different spatial slice if the HRRT surface is connected. However, since we are considering the holographic slice being built up using HRRT surfaces anchored to infinitesimal subregions, those anchored on both sides always stay disconnected.

Thus, the holographic slice, as seen in Fig. 4, is the union of static slices in both exterior regions and terminates at the bifurcation surface. As shown in Ref. [24], the bifurcation surface itself is extremal and lies on a Killing horizon, and hence the process of renormalizing leaves must asymptote to this surface.

The phenomenon of a holographic screen being terminated at a nontrivial surface requires the existence of a bifurcation surface, which is absent in most physical situations. For example, consider an AdS-Vaidya metric

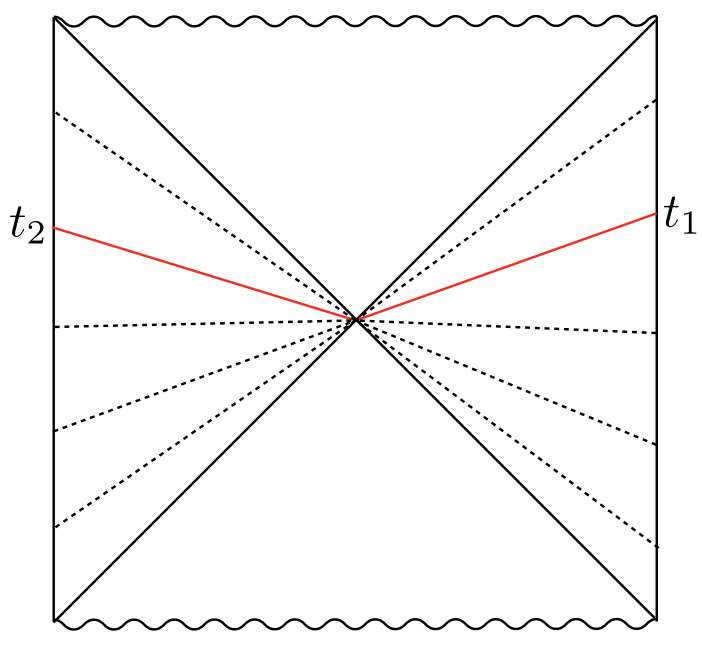

FIG. 4. The exterior of a two-sided eternal AdS black hole can be foliated by static slices (black dotted lines). The holographic slice (red) connects the boundary time slices at $t=t_{1}$ on the right boundary and $t=t_{2}$ on the left boundary to the bifurcation surface along these static slices.

where a black hole is formed from the collapse of a thin null shell of energy [52]. The metric in ingoing EddingtonFinkelstein coordinates is given by

$$
d s^{2}=-f(r, v) d v^{2}+2 d v d r+r^{2} d \Omega_{d-1}^{2},
$$

where

$$
f(r, v)=1+\frac{r^{2}}{L^{2}}-\theta(v)\left(\frac{r_{+}}{r}\right)^{d-2}\left(1+\frac{r_{+}^{2}}{L^{2}}\right),
$$

with

$$
\theta(v)= \begin{cases}0 & \text { for } v<0 \\ 1 & \text { for } v>0\end{cases}
$$

The null shell lies at $v=0$, and this spacetime is obtained simply by stitching together an AdS-Schwarzschild metric to the future of the shell and a pure AdS metric to the past. The composite global spacetime is time dependent, but each of the building blocks admits a timelike Killing vector locally as shown in Fig. 5. As discussed earlier, since the HRRT surfaces relevant to the holographic slice are those of infinitesimal subregions, they only sense the local spacetime, which is static. This allows us to construct the holographic slice independently in each region. The static slices can then be stitched together to obtain the holographic slice as shown in Fig. 5.

An important feature here is that at late times, i.e., sufficiently after the black hole has stabilized, the holographic slice constructed from a leaf stays near the horizon for a long time. Eventually, this flow terminates at $r=0$. 


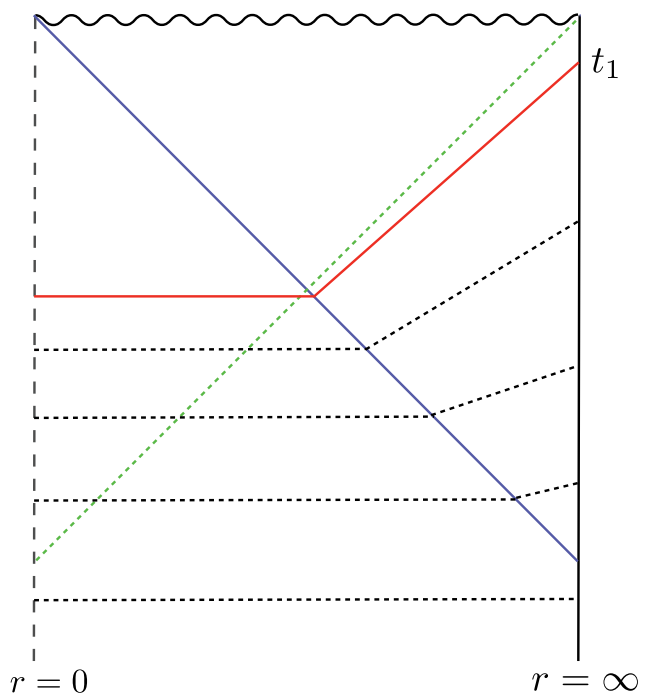

FIG. 5. Penrose diagram of an AdS-Vaidya spacetime formed from the collapse of a null shell (blue), resulting in the formation of an event horizon (green). Individual portions of the spacetime, the future and past of the null shell, are static. Thus, the holographic slice (red) can be constructed by stitching together a static slice in each portion.

This behavior of holographic slices is, in fact, general in one-sided black holes; see Fig. 6 for a schematic depiction.

Note that the picture of Fig. 6 is obtained in the $G_{\mathrm{N}} \rightarrow 0$ limit. When $G_{\mathrm{N}} \neq 0$, renormalized leaves will hit the stretched horizon [53], where the semiclassical description of spacetime breaks down, before being subjected to the long flow near the horizon.

\section{Friedmann-Robertson-Walker spacetimes}

We now discuss a nontrivial example in a time-dependent spacetime, away from the standard asymptotically AdS context. Consider a $(d+1)$-dimensional flat FriedmannRobertson-Walker (FRW) spacetime containing a single fluid component with equation-of-state parameter $w$

$$
d s^{2}=a(\eta)^{2}\left(-d \eta^{2}+d r^{2}+r^{2} d \Omega_{d-1}^{2}\right) .
$$

Here,

$$
a(\eta)=c|\eta|^{q},
$$

where $c>0$ is a constant, and

$$
q=\frac{2}{d-2+d w} .
$$

The discontinuity of $q$ at $w=(2-d) / d$ is an artifact of choosing conformal time, and physics is smooth across this value of $w$.

The spherically symmetric holographic screen is located at

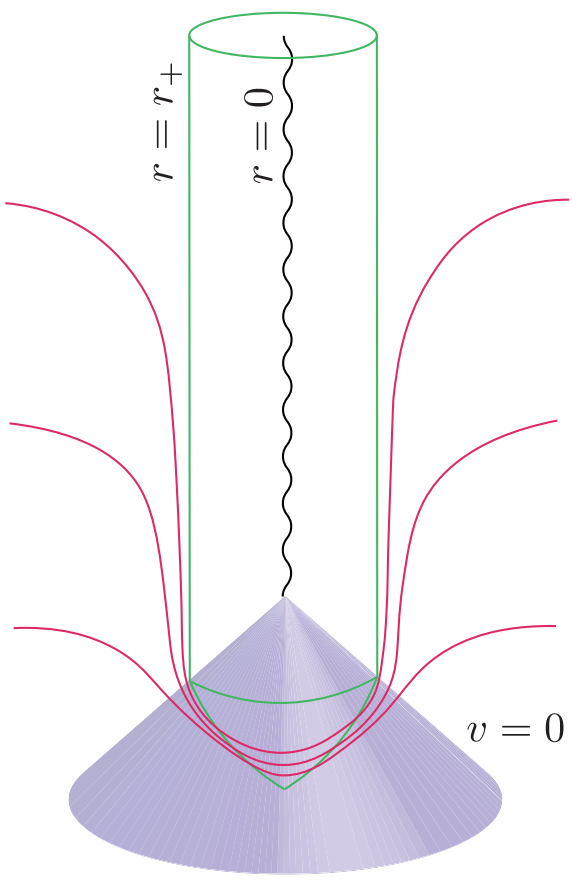

FIG. 6. A schematic depiction of holographic slices for a spacetime with a collapse-formed black hole in ingoing Eddington-Finkelstein coordinates.

$$
r(\eta)=\frac{a(\eta)}{\frac{d a}{d \eta}(\eta)}=\frac{\eta}{q}
$$

By spherical symmetry, the holographic slice must be a codimension-1 surface of the form $\eta=g(r)$, where each renormalized leaf is an $\mathbb{S}^{d-1}$. Consider a renormalized leaf at $\eta=\eta_{*}$ and $r=r_{*}$. Generalizing the results from Refs. [24,54], HRRT surfaces anchored to a small spherical cap of half-opening angle $\gamma$ of the renormalized leaf are given by

$$
\eta(\xi)=\eta_{*}+\frac{\dot{a}}{2 a}\left(\xi_{*}^{2}-\xi^{2}\right)+\cdots,
$$

where $\xi=r \sin \theta$ and $\xi_{*}=r_{*} \sin \gamma$ with $\theta$ being the polar angle, and $a \equiv a\left(\eta_{*}\right)$ and $\dot{a} \equiv d a / d \eta\left(\eta_{*}\right)$. We refer the reader to Appendix C 3 of Ref. [24] for more details.

The next renormalized leaf is generated by joining together the deepest point of each such HRRT surface. Suppose $\Delta \eta$ and $\Delta r$ represent the change in conformal time and radius from one renormalized leaf to the next. Then we have

$$
\begin{aligned}
\Delta \eta & =\frac{\dot{a}}{2 a} \xi_{*}^{2}+\cdots, \\
\Delta r & =-\left(r_{*}-r_{*} \cos \gamma\right),
\end{aligned}
$$

so that 


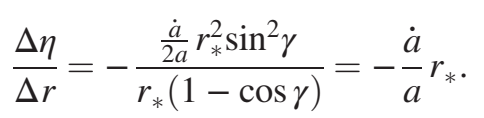

Taking the limit $\gamma \rightarrow 0$, we obtain a differential equation for the radial evolution of the holographic slice

$$
\frac{d \eta}{d r}=-\frac{q r}{\eta} .
$$

Integrating this equation gives us

$$
\eta^{2}+q r^{2}=\eta_{*}^{2}+q r_{*}^{2}=\frac{1+q}{q} \eta_{0}^{2}
$$

where $\eta_{0}$ is the conformal time of the original nonrenormalized leaf.

Let us highlight a few interesting features of this holographic slice. First, it spans the entire interior region of the holographic screen. Next, substituting $r=\eta / q$ into Eq. (22) tells us that the holographic slice starts out in the null direction from the leaf; see Eq. (17). This is because the $k$ direction locally has zero expansion there, as discussed in Sec. III B. As we move inward along the radial flow, however, the slope becomes flatter, and eventually the surface reaches the highest point given by

$$
\eta(r=0)=\eta_{0} \sqrt{\frac{1+q}{q}} .
$$

In Fig. 7 we have depicted holographic slices, given by Eq. (23), for several values of $w$ with $d=3$.

\section{Asymptotically AdS and flat spacetimes}

Here we discuss certain subtleties associated with holographic screens that lie on an asymptotic boundary. First, consider a $(d+1)$-dimensional asymptotically AdS spacetime, which can be expanded in a Fefferman-Graham series [55] near the boundary

$$
d s^{2}=\frac{L^{2}}{z^{2}}\left\{g_{a b}\left(x^{a}, z\right) d x^{a} d x^{b}+d z^{2}\right\}
$$

where $L$ is the AdS length scale, and

$$
g_{a b}\left(x^{a}, z\right)=g_{a b}^{(0)}\left(x^{a}\right)+z^{2} g_{a b}^{(2)}\left(x^{a}\right)+\cdots
$$

Here, $g_{a b}^{(0)}$ represents the conformal boundary metric, and the subleading corrections represent deviations as one moves away from the boundary at $z=0$.

In an asymptotically AdS spacetime, the holographic screen $H$ formally lies at spacelike infinity. In order to construct a holographic slice in such a situation, one needs to first consider a regularized screen $H^{\prime}$ at $z=\epsilon$ and then take the limit $\epsilon \rightarrow 0$ after constructing the slice. Suppose

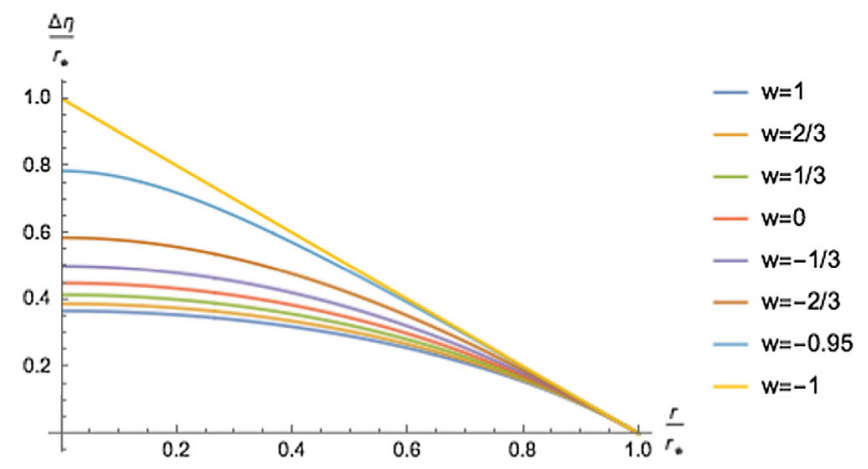

FIG. 7. Holographic slices of $(3+1)$-dimensional flat FRW universes containing a single fluid component with equation-ofstate parameter $w$.

that a leaf is given by a constant $t$ slice of $H^{\prime}$, with $h_{i j}$ representing the induced metric on the leaf. The null normals are then given by

$$
\begin{aligned}
& k_{\mu}=d z-d t+O\left(\epsilon^{2}\right), \\
& l_{\mu}=-d z-d t+O\left(\epsilon^{2}\right),
\end{aligned}
$$

where the $O\left(\epsilon^{2}\right)$ corrections arise due to deviations away from the boundary. The null expansions are

$$
\begin{aligned}
& \theta_{k}=h^{i j} \Gamma_{i j}^{z}-h^{i j} \Gamma_{i j}^{t}=-\frac{\epsilon(d-1)}{L}+O\left(\epsilon^{2}\right), \\
& \theta_{l}=-h^{i j} \Gamma_{i j}^{z}-h^{i j} \Gamma_{i j}^{t}=\frac{\epsilon(d-1)}{L}+O\left(\epsilon^{2}\right) .
\end{aligned}
$$

Thus, we see that the expansion $\theta_{k}$ vanishes only in the limit $\epsilon \rightarrow 0$.

This implies that a leaf $\sigma^{\prime}$ of a regularized screen $H^{\prime}$ $(\epsilon \neq 0)$ is, in fact, a renormalized leaf $\left(\theta_{k} \neq 0\right)$, and thus the results in Sec. III B-that the holographic slice initially extends in the null direction and the leaf area remains constant-do not apply. In fact, the holographic slice extending from $\sigma^{\prime}$ initially evolves inward along the $z$ direction up to corrections of $O(\epsilon)$, as can be seen from the fact that $\theta_{k}=-\theta_{l}$ up to $O(\epsilon)$. In the limit $\epsilon \rightarrow 0$, both $\theta_{k}$ and $\theta_{l}$ vanish simultaneously. This leads to a holographic screen at spacelike infinity in a formal sense. ${ }^{7}$

A similar situation arises in asymptotically flat spacetimes. A general asymptotically flat spacetime can be expanded in the Bondi-Sachs form [56,57] as

\footnotetext{
${ }^{7}$ Strictly speaking, this does not satisfy the definition of the holographic screen in Sec. III A, which requires $\theta_{l}$ to be strictly positive.
} 


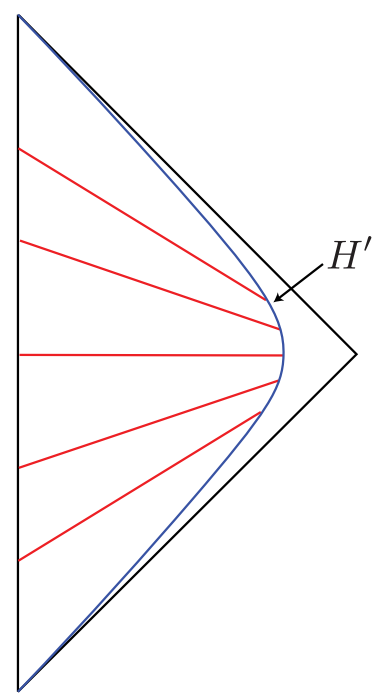

FIG. 8. Penrose diagram of a Minkowski spacetime. The holographic slices (red) are anchored to the regularized holographic screen $H^{\prime}$ (blue). As the limit $R \rightarrow \infty$ is taken, the holographic slices become complete Cauchy slices.

$$
\begin{aligned}
d s^{2}= & -\frac{V}{r} e^{2 \beta} d u^{2}-2 e^{2 \beta} d u d r \\
& +r^{2} h_{A B}\left(d x^{A}-U^{A} d u\right)\left(d x^{B}-U^{B} d u\right),
\end{aligned}
$$

where each of the functions admits a large- $r$ expansion with the following behavior:

$$
\begin{aligned}
V & =r+O(1), & \beta & =O\left(\frac{1}{r^{2}}\right), \\
U^{A} & =O\left(\frac{1}{r^{2}}\right), & h_{A B} & =O(1) .
\end{aligned}
$$

In order to construct a holographic slice, the holographic screen $H$ must be regularized to become a timelike surface $H^{\prime}$ at $r=R$, where we can eventually take the limit $R \rightarrow \infty$. The null normals of a leaf on a constant time slice are

$$
\begin{aligned}
k_{\mu} & =d u, \\
l_{\mu} & =-\frac{V}{r} d u-2 d r,
\end{aligned}
$$

giving the null expansions near the boundary

$$
\begin{gathered}
\theta_{k}=-\frac{2}{R}+O\left(\frac{1}{R^{2}}\right), \\
\theta_{l}=\frac{2}{R}+O\left(\frac{1}{R^{2}}\right) .
\end{gathered}
$$

Thus, similar to the case of asymptotically AdS spacetimes, a leaf of a regularized holographic screen is a renormalized one, and both $\theta_{k}, \theta_{l} \rightarrow 0$ simultaneously as $R \rightarrow \infty$.
As a simple example, we illustrate the case of a Minkowski spacetime in Fig. 8. As the limit $R \rightarrow \infty$ is taken, the holographic slices become complete Cauchy hypersurfaces which are constant time slices anchored to spatial infinity. In the limit $t \rightarrow+\infty(-\infty)$, future (past) null and timelike infinities are obtained as a holographic slice. In this situation, time evolution of the boundary theory from $t \rightarrow-\infty$ to $+\infty$ corresponds to an $S$-matrix description of the bulk.

\section{INTERPRETATION AND APPLICATIONS}

We have introduced the geometric definition of the holographic slice and demonstrated some of its properties. But what does the slice correspond to in the boundary theory? What questions can it help us address? The construction of the slice naturally lends itself to an interpretation of eliminating information at small scales, and hence can be well understood in the context of coarse graining. Through this, we can think of the slice as an isometric tensor network. This provides us with a new way to think about holographic tensor networks and the bulk regions of spacetime that they encode.

Throughout this section we will be talking about various Hilbert spaces in which holographic states belong. To do so, we will be taking $G_{\mathrm{N}}$ to be finite but small. This is an appropriate approximation for classical spacetimes provided we only concern ourselves with length scales sufficiently larger than the Planck length.

\section{A. Coarse graining}

We take the view that a boundary state, $|\psi(0)\rangle$, lives on the original leaf, $\Upsilon(0)$, i.e., it lives in an effective Hilbert space, $\mathcal{H}$, having a local product space structure with dimension $\log |\mathcal{H}|=\|\Upsilon(0)\| / 4 G_{\mathrm{N}}$. The HRRT prescription says that the emergent bulk geometry is intricately related to the entanglement of the boundary state. In particular, despite the fact that bulk information is delocalized in the boundary theory, a bulk region cannot be reconstructed if some boundary subregions are ignored. The size of the smallest subregion for this to occur gives us some idea of what scale of boundary physics this bulk region is encoded in. Using this intuition, we can then attempt to address what coarse graining the boundary state corresponds to in the bulk.

At each step in the construction of the holographic slice, we eliminate the region of spacetime associated with a small length scale, $\delta$, of the boundary. In particular, this is the region of spacetime whose information is necessarily lost if we cannot resolve below the length scale $\delta$. In this sense, we are coarse graining over the scale $\delta$ and obtaining a new bulk region whose information has not been lost. Recursively doing this and sending $\delta$ to zero produces a continuum of bulk domains of dependence with unique 


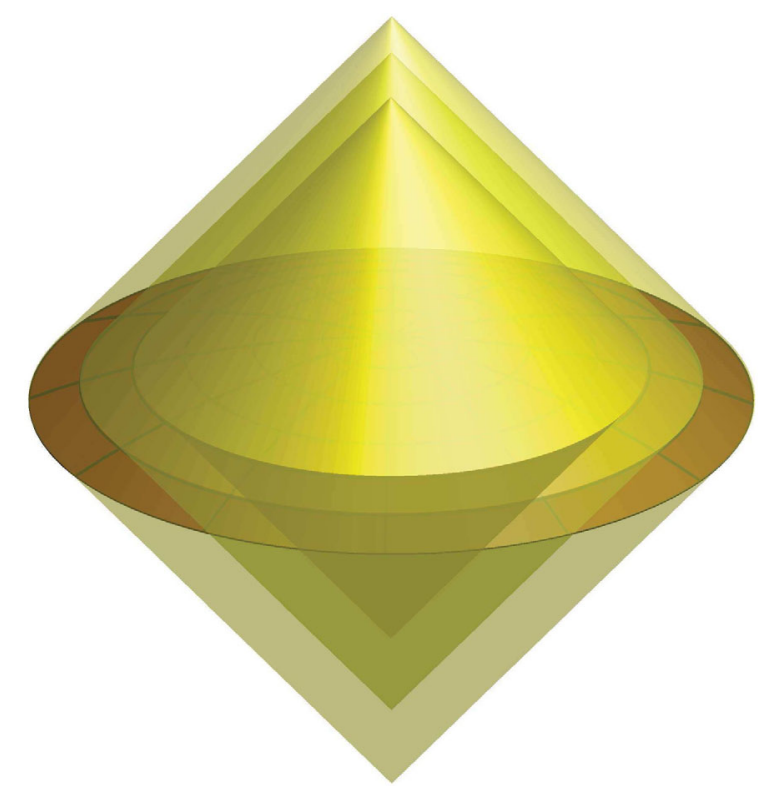

FIG. 9. This depicts the holographic slice (maroon), and the successive domains of dependence encoded on each renormalized leaf.

boundaries sweeping out the holographic slice, $\Upsilon(\lambda)$. This is depicted in Fig. 9.

A consistency check for this interpretation is that the size of the effective Hilbert space should necessarily decrease as we coarse grain over larger and larger scales. This is precisely the monotonicity property listed in Sec. III: as one flows along the holographic slice, the area of the renormalized leaves decreases. This tells us that the size of the effective Hilbert space describing the bulk domain of dependence also decreases.

As the coarse-graining procedure progressively removes information at small scales, a corresponding removal of bulk information closest to the renormalized leaf occurs. Given this global removal of short-range information, one should expect the entanglement between any region and its complement to correspondingly decrease. This is precisely the monotonicity of entanglement entropy property observed in Sec. III. This is consistent with the interpretation that at each step we are removing short-range entanglement.

Using the holographic slice, we can address the question of how much entanglement between a subregion and its complement is sourced by physics at different scales. By following the integral curves of $s$ for the subregion, we can stop at whatever scale we desire and use the HRRT prescription on the renormalized leaf. This gives us the entanglement entropy sourced by physics at length scales larger than that associated with the renormalized leaf.

\section{B. Radial evolution of states}

We will now be more explicit in describing the framework for coarse graining holographic states. Given a bulk region $D(\Upsilon(0))$, there exists a quantum state $|\psi(0)\rangle$ living in some fundamental holographic Hilbert space, $\mathcal{H}_{\mathrm{UV}}$, in which the bulk information of $D(\Upsilon(0))$ is encoded via the HRRT prescription. This implies that $\mathcal{H}_{\mathrm{UV}}$ has a locally factorizable structure. On the other hand, the area of $\Upsilon(0)$ provides an upper bound for the dimension of the effective Hilbert space that $|\psi(0)\rangle$ lives in, which we call $\mathcal{H}_{\Upsilon(0)}$. That is, $|\psi(0)\rangle \in \mathcal{H}_{\Upsilon(0)} \subset \mathcal{H}_{\mathrm{UV}}$. The dimension, $\left|\mathcal{H}_{\Upsilon(0)}\right|$, of the effective Hilbert space is defined as

$$
\ln \left|\mathcal{H}_{\Upsilon(0)}\right|=\sum_{i} S_{i}=\frac{\|\Upsilon(0)\|}{4 G_{\mathrm{N}}}
$$

Here, $S_{i}$ represents the entanglement entropy of $|\psi(0)\rangle$ in an infinitesimally small subregion, $A_{i}$, of the holographic space $\Omega$ on which $\mathcal{H}_{\mathrm{UV}}$ is defined. We sum over all of these small subregions such that $\Omega=\cup_{i} A_{i}$ and $A_{i} \cap A_{j}=\varnothing$ $(i \neq j)$. This reduces to calculating the area of $\Upsilon(0)$ because of the HRRT prescription. Namely, the size of the effective Hilbert space that $|\psi(0)\rangle$ lives in is determined by the entanglement between the fundamental d.o.f. of $\mathcal{H}_{\mathrm{UV}}$, and $\|\Upsilon(0)\| / 4 G_{\mathrm{N}}$ is the thermodynamic entropy associated with this entanglement structure.

As one continuously coarse grains $|\psi(0)\rangle$, information encoded in small scales is lost. Correspondingly, information of the bulk geometry that is stored in small scales is lost, and the dimension of the effective Hilbert space that the coarse-grained state lives in decreases. At a given scale of coarse graining corresponding to $\lambda$, the new state, $|\psi(\lambda)\rangle$, lives in the same Hilbert space as the original leaf, $\mathcal{H}_{\mathrm{UV}}$, but now in an effective subspace, $\mathcal{H}_{\Upsilon(\lambda)}$, with dimension given by $\ln \left|\mathcal{H}_{\Upsilon(\lambda)}\right|=\|\Upsilon(\lambda)\| / 4 G_{\mathrm{N}}$. Additionally, $|\psi(\lambda)\rangle$ only contains information of $D(\Upsilon(\lambda))$, as we have explicitly lost the information necessary to reconstruct any part of $D(\Upsilon(0)) \backslash D(\Upsilon(\lambda))$.

Because all of the coarse-grained states live in the same Hilbert space, $\mathcal{H}_{\mathrm{UV}}$, we can consider the coarsegraining procedure as a unitary operation that takes us from state to state, along the lines of the work in Ref. [58]. That is,

$$
|\psi(\lambda)\rangle=U(\lambda, 0)|\psi(0)\rangle
$$

We can write $U\left(\lambda_{1}, \lambda_{2}\right)$ as

$$
U\left(\lambda_{1}, \lambda_{2}\right)=P \exp \left[-i \int_{\lambda_{2}}^{\lambda_{1}} K(\lambda) d \lambda\right]
$$

where $P$ represents path ordering. $K(\lambda)$ is a Hermitian operator removing physical correlations between nearby subregions at the length scale, $l_{\lambda}$, associated to $\lambda$ on $\Omega$. Some appropriate measures of physical correlations would be the mutual information $[I(A, B)]$, entanglement negativity $[N(A, B)]$, or entanglement of purification $[E(A, B)]$ 
between neighboring small subregions of the leaf under consideration, $\Upsilon(\lambda)$. Note that as the boundary state becomes maximally entangled, all three of these measures will vanish. This also happens if the boundary state in consideration has no entanglement, i.e., is a product state.

Of the three measures, entanglement of purification already has a bulk description that naturally characterizes some measure of moving into the bulk. In particular, the authors of Refs. $[59,60]$ proposed that the entanglement of purification of two boundary subregions, $A$ and $B$, is calculated by the minimum cross section, $\zeta$, of a bipartition of the extremal surface anchored to $A \cup B$; see Fig. 10. Considering the case where $\partial A$ and $\partial B$ coincide at some point, and whose connected phase is the appropriate extremal surface, the entanglement of purification gives some measure of the depth of the extremal surface. In bulk dimensions higher than $2+1,\|\zeta\|$ will not be in units of length, but it is still related to the depth of the extremal surface. Thus it seems natural that $K(\lambda)$ is some (quasi) local function, $F$, of physical correlations, including but not necessarily limited to quantum entanglement, at the scale $l_{\lambda}$. For example, it may be related to the entanglement of purification:

$$
K(\lambda) \sim \int d^{d-1} \mathbf{x} F\left(E_{\lambda}(\mathbf{x})\right)
$$

where $\mathbf{x}$ are the coordinates of $\Omega$. Here, $E_{\lambda}(\mathbf{x})$ is the "density" of the entanglement of purification between the d.o.f. in two neighboring regions on $\sigma(\lambda)$ around $\mathbf{x}$.

Alternately, the case of subregion coarse graining as in Fig. 2 motivates the usage of results from Ref. [61], which can be used to map the state from $\sigma$ to $\sigma(\lambda)$. In AdS/CFT, modular evolution allows one to explicitly reconstruct bulk operators on the HRRT surface. With our assumption that the HRRT formula holds (with quantum corrections), a similar construction should be possible given complete knowledge of the boundary theory. $K(\lambda)$ may then be better understood as a convolution over modular evolutions with infinitesimal boundary subregions:

$$
K(\lambda) \sim \int d^{d-1} \mathbf{x} F\left(\kappa_{\lambda}(\mathbf{x})\right)
$$

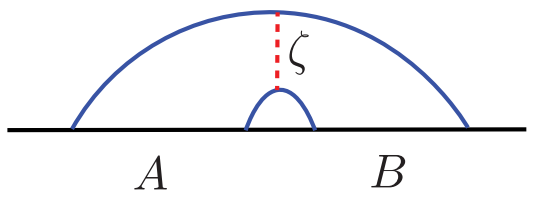

FIG. 10. Let $A$ and $B$ two boundary subregions. The blue lines represent the HRRT surface of $A \cup B$ and $\zeta$ is the minimal cross section. The entanglement of purification of $A$ and $B$ is given by $\|\zeta\| / 4 G_{\mathrm{N}}$. In the limit that $A$ and $B$ share a boundary point, $\zeta$ probes the depth of the extremal surface. where $\kappa_{\lambda}(\mathbf{x})$ is the modular Hamiltonian density on $\sigma(\lambda)$ at $\mathbf{x}$. It would be interesting to make the connection of $K(\lambda)$ to modular evolution clearer in the future.

The process of removing short-range correlations continues until all correlations at the scale $l_{\lambda}$ have been removed, and hence no more bulk spacetime can be reconstructed. This can happen when the slice contracts to a point and no local product structure exists in the effective Hilbert space $\mathcal{H}_{\Upsilon(\lambda)}$. Note that in $\mathcal{H}_{\mathrm{UV}}$ this state corresponds to a product state, so that $S_{i}=0$ for every subregion in $\mathcal{H}_{\mathrm{UV}}$. The other way in which all relevant correlations vanish is when the coarse-grained state becomes maximally entangled in $\mathcal{H}_{\Upsilon(\lambda)}$. In $\mathcal{H}_{\mathrm{UV}}$, this corresponds to a state which satisfies $S_{A}=\sum_{i \subset A} S_{i}$ for all subregions, $A$, of $\Omega$, where $i$ is a partition of $A$.

When the coarse-grained state becomes maximally entangled in $\mathcal{H}_{\Upsilon(\lambda)}, U(\lambda+d \lambda, \lambda)$ becomes the identity for Eq. (41) as $K(\lambda)$ becomes 0 . Hence, the state remains invariant under the coarse-graining operation. There are two ways for this to happen geometrically. One is if the slice approaches a bifurcation surface; then the extremal surfaces coincide with the renormalized leaf, hence preventing any further movement into the bulk. This is the case for eternal two-sided black holes. The second is if the slice approaches a null, nonexpanding horizon and the state is identical along the horizon. This is the case in de Sitter space. This result is complementary to Theorem 1 of Ref. [24], which proves that if a boundary state is maximally entangled, it must either live on a bifurcation surface or a null, nonexpanding horizon.

This may initially seem like a contradiction-that both the state becomes maximally entangled and that there are no more correlations to harvest. However, it is precisely because we are examining correlations at small scales that this occurs. A small boundary region is maximally entangled with the rest of the boundary, and hence the short-range entanglement must vanish. One can quantify this by examining the entanglement negativity of bipartitions of small subregions on $\sigma(\lambda)$. As states become maximally entangled, the entanglement negativity vanishes for two small subregions. This places an upper bound on the real, distillable entanglement between these subregions. Hence, the true quantum entanglement at small scales vanishes as a state becomes maximally entangled. Correspondingly, the coarse-graining procedure halts. This is indeed what happens to the holographic slice.

The same can also be seen by considering the modular evolution, Eq. (42), of a maximally entangled subregion. In this case, the modular evolution is proportional to the identity operator, and hence the modular flow of the state is stationary. This corresponds to no movement into the bulk as expected by the properties of the holographic slice for maximally entangled states. 


\section{Tensor network picture}

In this language the relationship to tensor networks is very clear. The holographic slice arises as the continuous limit of a tensor network that takes a boundary state and disentangles below a certain scale, reducing the effective Hilbert space size. This is a slight generalization of the continuous multiscale entanglement renormalization ansatz (cMERA) [27], which is restricted to hyperbolic geometries.

In general, we can consider a tensor network as a noncontinuum modeling of the holographic slice, which isometrically embeds boundary states into spaces of lower effective dimension by removing short-range correlations; see Fig. 11. In the ground state of $\mathrm{AdS} / \mathrm{CFT}$, this corresponds to an instance of the multiscale entanglement renormalization ansatz (MERA) [62]. Each layer of the tensor network then lives on the corresponding renormalized leaf of the discrete version of $\Upsilon$. From this we see that the tensor network lives on this discrete version of the holographic slice. Because isometric tensor networks obey a form of HRRT, one may mistakenly conclude that the cut

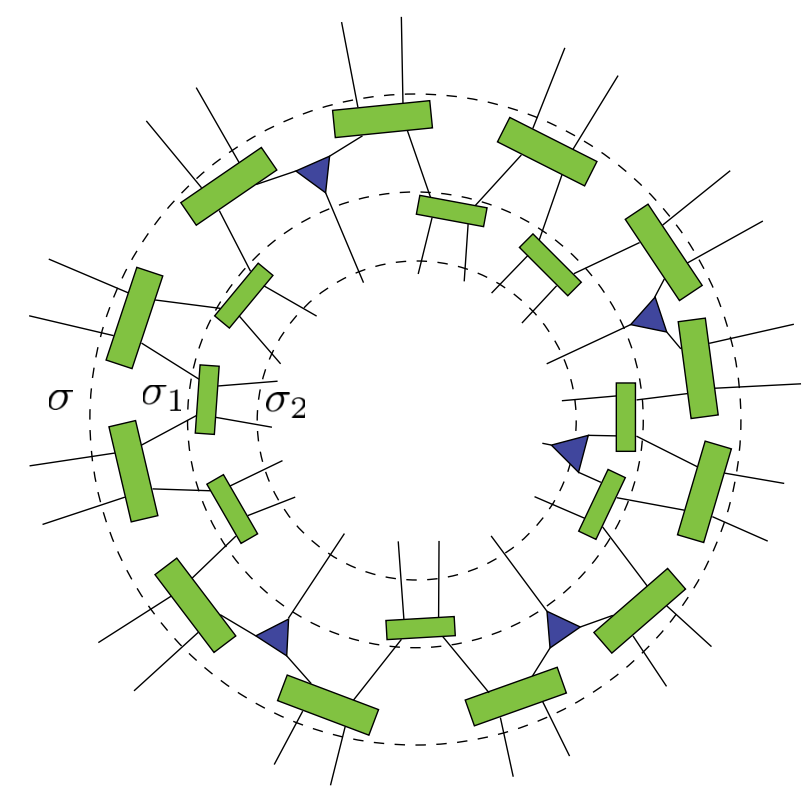

FIG. 11. A tensor network for a nonhyperbolic geometry. The green rectangles correspond to disentanglers while the blue triangles are coarse-graining isometries. Each internal leg of the tensor network has the same bond dimension. We are imagining that $\sigma$ corresponds to a leaf of a holographic screen and each successive layer $\left(\sigma_{1}\right.$ and $\left.\sigma_{2}\right)$ is a finite size coarsegraining step of the holographic slice. Through this interpretation, the tensor network lives on the holographic slice. However, the entanglement entropy calculated via the min-cut method in the network does not correspond to the distance of the cut along the holographic slice in the bulk. It corresponds to the HRRT surface in the appropriate domain of dependence. The locations of $\sigma_{1}$ and $\sigma_{2}$ in the bulk are found by convolving the HRRT surfaces for the small regions being disentangled and coarse grained. The holographic slice is a continuous version of this tensor network. through the network computes the area of the corresponding surface in the bulk along the holographic slice. This is generally not the case; the maximin method tells us that this area provides only a lower bound on the entanglement. The entanglement calculated in this way instead corresponds to the area of the HRRT surface anchored to the appropriate subregion of a renormalized leaf. In other words, the tensor network should not be viewed as a discretization of the holographic slice, but rather as a set of boundary states dual to successively smaller domains of dependence.

In fact, this interpretation can be applied to any isometric tensor network, and we argue that this is the proper way to view tensor networks representing bulk spacetimes. That is, given a state represented by an isometric tensor network, one can find a set of states by pushing the boundary state through the tensors one layer at a time such that no two layers have the same boundary legs. ${ }^{8}$ These successive states are then dual to bulk domains of dependence that are successively contained in each other, and whose boundaries lie on (a discrete version of) the holographic slice.

\section{Time evolution and gauge fixing}

The preferred holographic slice of Sec. III B 1 provides us with a novel way to foliate spacetimes. By applying the holographic slice procedure to each boundary time slice, one foliates the bulk spacetime with holographic slices.

In order for this to provide a good gauge fixing, the holographic slices generated from different boundary time slices must not intersect. In spherically symmetric cases, these intersections do not occur. From the spherical symmetry of the spacetime, the renormalized leaves must also be spherical. Thus, if two holographic slices did intersect, they must intersect at a renormalized leaf. However, the evolution of the slice is unique from this leaf, and hence these two slices do not intersect. Furthermore, the reverse flow is also unique and hence the slices must exactly coincide. This prevents ambiguities in the gauge fixing of the bulk spacetime.

Outside of spherically symmetric cases, if $\operatorname{sgn}(K)$, the sign of the extrinsic curvature of the slice, is constant over the slice then no slices will intersect. By joining a slice with the past (future) portion of the holographic screen in the $K \geq 0(\leq 0)$ case, one can create a barrier for extremal surfaces anchored in the interior of the barrier. This implies that any slice constructed from a leaf cannot penetrate

\footnotetext{
${ }^{8}$ This picture can perhaps be used to show that the dynamical holographic entropy cone is contained within the holographic entropy cone [14]. By explicitly constructing the holographic slice tensor network that encodes a state of a time dependent geometry, we will have found a model that encodes the entropies in a way that ensures containment within the holographic entropy cone.
} 
slices generated from leaves towards its future (past) if $K \geq 0(\leq 0)$.

As explained in Secs. III B and IV, the foliation generated by the holographic slices will not probe behind latetime horizons. Thus this foliation provides a gauge fixing of the region of spacetime exterior to black hole horizons. In this region, the foliation provides a covariant map from boundary time slices to bulk time slices.

\section{RELATIONSHIP TO RENORMALIZATION}

In this section, we discuss how our coarse-graining procedure is related to conventional renormalization, both in the context of standard quantum field theories and AdS/CFT.

\section{A. Analogy to renormalization in quantum field theories}

We first draw an analogy between pulling in the boundary along the holographic slice and standard renormalization in quantum field theories. In particular, we liken the limitations of fixed-order perturbation theory to the existence of reconstructable shadows. We begin by reviewing renormalization in quantum field theories phrased in a way to make the relationship clear.

Suppose one computes the amplitude of a process involving two widely separated mass scales $m$ and $E$ in fixed-order perturbation theory. In terms of a renormalized coupling constant $g$, it is given generally in the form

$$
\mathcal{M}=\sum_{n=0}^{\infty} c_{n}\left(\frac{g}{16 \pi^{2}} \ln \frac{E}{m}\right)^{n},
$$

where the $c_{n}$ 's are of the same order. This implies that even if $g / 16 \pi^{2}$ is small, this perturbation theory breaks down when $\ln (E / m) \sim 16 \pi^{2} / g$.

There is, however, a way to resum these logarithms: the renormalization group. Introducing the concept of a running coupling constant, $g(\mu)$, defined at a sliding scale $\mu$, the amplitude of Eq. (43) can be written as

$$
\mathcal{M}=\sum_{n=0}^{\infty} c_{n}\left(\frac{g(\mu)}{16 \pi^{2}} \ln \frac{E}{\mu}\right)^{n} .
$$

The process can now be calculated perturbatively as long as both $g(m) / 16 \pi^{2}$ and $g(E) / 16 \pi^{2}$ are small, where $g(m)$ and $g(E)$ are related by a continuous renormalization group evolution. In general, the range of validity of this renormalization-group-improved perturbation theory is larger than that of fixed-order perturbation theory.

This phenomenon is analogous to the existence of shadows in the holographic reconstruction. If one tries to reconstruct the bulk in a "single shot" using HRRT surfaces anchored to the original leaf, then there can be regions in spacetime (shadows) that cannot be reconstructed. This, however, is not a fundamental limitation of the perturbative reconstruction of the bulk. As we have seen, we can reconstruct a portion of entanglement shadows by performing a reconstruction in multiple steps by first renormalizing the leaf and then using HRRT surfaces anchored to the renormalized leaf. By doing this renormalization with more steps, one can progressively probe deeper into shadow regions. Going to the continuum limit (the holographic slice), we find that we can describe physics in shadows without difficulty.

Even with the renormalization group improvement, the perturbative description of physics stops working when $g(\mu)$ hits a Landau pole or approaches a strongly coupled fixed point. This is analogous to the fact that the evolution of the holographic slice halts, $U\left(\lambda_{1}, \lambda_{2}\right) \propto \mathbb{1}$ in Eq. (40), once the renormalized leaf contracts to a point or approaches a horizon. Incidentally, this picture is consonant with the idea that describing the interior of a black hole would require "nonperturbative" physics. ${ }^{9}$

We stress that from the boundary point of view, the renormalization of a leaf corresponds to the coarse graining of a state at a fixed time. A natural question is if there is an effective theory relating coarse-grained states at different times. We do not see a reason to doubt the existence of such a theory, at least for d.o.f. sufficiently deep in the bulk. Since the coarse graining depends on the state, however, the resulting description may well be applicable only within a given geometry, i.e., a selected semiclassical branch of the fundamental state in quantum gravity.

\section{B. Comparison to holographic renormalization in AdS/CFT}

How is the holographic slice related to holographic renormalization in asymptotically AdS spacetimes? There is extensive literature devoted to the latter subject. Here we will highlight the essential difference between our renormalization procedure and the perspective of Susskind and Witten [67].

If one kept $N^{2}$ (the number of field d.o.f.) fixed per cutoff cell and applied the Susskind-Witten method of regularization deeper in the bulk, a local description of physics on the boundary would break down once $R \approx l_{\mathrm{AdS}}$. Here, $R$ and $l_{\mathrm{AdS}}$ are the radius of the cutoff surface and the AdS length scale, respectively. This is because the number of d.o.f. within an $l_{\mathrm{AdS}}$-sized bulk region is of order $\left(l_{\mathrm{AdS}} / l_{\mathrm{P}}\right)^{d-1}$, which is just $N^{2}$. Here, $l_{\mathrm{P}}$ is the Planck length in the bulk.

However, holography extends to sub-AdS scales, and the extremal surfaces anchored to a cutoff at $l_{\text {Ads }}$ satisfy the appropriate properties to be interpreted as entanglement entropies $[13,15]$. As emphasized throughout the

\footnotetext{
${ }^{9}$ In particular, an interior description may require changing the basis of multiple black hole microstates [63,64], each of which can be viewed as having different background geometries corresponding to slightly different black hole masses $[65,66]$.
} 
text, the connection between entanglement and geometric quantities seems to extend beyond AdS/CFT $[19,68]$. Because of this, we expect that there should be some way to renormalize the boundary state in such a way to preserve the HRRT prescription at all scales. This is, however, prohibited if we fix $N^{2}$ because already at an $l_{\text {AdS }}$-sized region we lose the ability to talk about the entanglement of boundary subregions (as there is only one boundary cell).

Therefore, if one wants to preserve the ability to use the HRRT prescription, $N^{2}$ must change as the boundary is pulled in. Simply requiring that $N^{2} \geq 1$ per cell will allow renormalization down to $l_{\mathrm{P}}$. This is easily seen by noticing that the number of cells for a boundary moved in to radius $R\left(\leq l_{\mathrm{AdS}}\right)$ is given by the total number of bulk d.o.f., $\left(R / l_{\mathrm{P}}\right)^{d-1}$, divided by $N^{2}$. This implies that when $R=l_{\mathrm{P}}$ the number of cells is of order unity, and the holographic description must break down. In fact, the bulk description is expected to break down before this happens. Suppose that the gauge coupling, $g$, of the boundary theory stays constant. Then the requirement of a large 't Hooft coupling, $N^{2} \geq 1 / g^{4}=\left(l_{\mathrm{s}} / l_{\mathrm{P}}\right)^{d-1}$, implies that the bulk spacetime picture is invalidated when $R \lesssim l_{\mathrm{s}}$. Here, $l_{\mathrm{s}}$ is the string length. Assuming the existence of a renormalization scheme preserving the HRRT prescription implies that there exists a way to redistribute the original $N^{2}$ d.o.f. spatially on a coarse-grained holographic space.

The construction of the holographic slice requires extremal surfaces to be anchored to renormalized leaves, so the renormalization procedure utilized must necessarily preserve the ability to use the HRRT prescription. The holographic slice, therefore, must employ the special renormalization scheme described above.

\section{DISCUSSION}

The holographic slice is defined using HRRT surfaces, and hence is inherently background dependent. This prohibits the use of the holographic slice as some way to analyze the coarse-grained behavior of complex quantum-gravitational states with no clear bulk interpretation. In particular, if a state is given by a superposition of many different semiclassical geometries,

$$
|\Psi\rangle=c_{1}\left|\psi_{1}\right\rangle+c_{2}\left|\psi_{2}\right\rangle+\cdots,
$$

then the holographic slice prescription can be applied to each branch of the wave function, $\left|\psi_{i}\right\rangle$, independently. However, there is no well-defined slice for $|\Psi\rangle$. This is the same limitation one would face when considering the entanglement wedge of similar states. Despite this, for superpositions of states within the code subspace, the analyses of Refs. [23,69] tell us that the holographic slice construction is well defined.
Regardless, the holographic slice sheds light on the nature of bulk emergence. The construction of the slice harvests short-range entanglement between small subregions - not in the form of entanglement entropy. It is precisely this that allows the slice to flow into the bulk and through entanglement shadows. This work emphasizes the idea that entanglement entropy as measured by von Neumann entropy is not sufficient to characterize the existence of a semiclassical bulk viewed from the boundary. Other measures of entanglement (negativity, entanglement of purification, etc.) may be more useful to analyze bulk emergence. This was explored extensively in Ref. [24].

The slice additionally provides a very natural interpretation for nonminimal extremal surfaces as the entanglement entropy for subregions of coarse-grained states. Because the coarse-graining procedure mixes up the boundary d.o.f. while removing the short-range information, the interpretation of nonminimal extremal surfaces in terms of purely UV boundary terms will necessarily be very complicated [49]. However, once coarse graining occurs these complicated quantities manifest with a simple interpretation. This is also what is seen in the entanglement of purification calculations.

By assuming that the holographic states all live within the same infinite-dimensional Hilbert space, $\mathcal{H}_{\mathrm{UV}}$, we were able to discuss the mapping from a boundary state to a coarse-grained version of itself. This is what gave rise to the $K(\lambda)$ operator in Sec. V B. Alternatively, rather than use $\mathcal{H}_{\mathrm{UV}}$ to discuss coarse graining, one can use it to talk about time evolution in the boundary theory. One of the major hurdles in formulating theories for holographic screens is the fact that the area of the screens is nonconstant. If one were to view this area as determining the size of the true Hilbert space the state lived in, then time evolution would require transitions between Hilbert spaces. However, by viewing the leaf area as a measure of the size of the effective subspace that the state lives in, we are free from this complication. In fact, modeling time evolution is similar to performing the reverse of the coarse-graining operation. This introduces entanglement at shorter and shorter scales, which increases the effective subspace's size. Of course, time evolution must account for other complex dynamics, but simply increasing the screen area is not difficult. This interpretation suggests that the area of holographic screens is a thermodynamic entropy measure, rather than a measure of the fundamental Hilbert space size.

Concluding, the holographic slice is a novel, covariantly defined geometric object. It encodes the bulk regions dual to successively coarse-grained states and we proposed that the flow along the slice is governed by distillable correlations at the shortest scales. This may be related to the entanglement of purification of small regions or the modular evolution of such regions. An investigation of the explicit boundary flow along the slice seems to be the most promising avenue of future work. It may also be 
fruitful to study the mean curvature vector flow of codimension-2 convex surfaces in Lorentzian spacetimes, as characterizing solutions to this flow may provide insights into the coarse-graining operation.

\section{ACKNOWLEDGMENTS}

We thank Chris Akers, Ning Bao, Venkatesa Chandrasekaran, Grant Remmen, Fabio Sanches, and Arvin Shahbazi-Moghaddam for discussions. This work was supported in part by the National Science Foundation under grants PHY-1521446, by MEXT KAKENHI Grant No. 15H05895, and by the Department of Energy, Office of Science, Office of High Energy Physics, under Contract No. DE-AC02-05CH11231.

\section{APPENDIX A: INTERSECTION OF DOMAINS OF DEPENDENCE}

Lemma 1. Let $\Sigma$ be a closed, achronal set and $D(\Sigma)$ be the domain of dependence of $\Sigma$. Let $p$ and $q$ be points in $D(\Sigma)$, and $\lambda$ a causal curve such that $\lambda(0)=p$ and $\lambda(1)=$ $q$ where $p$ lies to the past of $q$. Then, all points $r=\lambda(t)$ for $t \in[0,1]$ are contained in $D(\Sigma)$.

Proof.-Suppose that such a point $r$ does not belong to $D(\Sigma)$. Then, there must exist an inextendible causal curve $\lambda^{\prime}$ that passes through $r$ and does not intersect $\Sigma$. Without loss of generality, assume that $r$ lies to the past of $\Sigma$. Consider a causal curve composed of $\lambda$ to the past of $r$ and $\lambda^{\prime}$ to the future of $r$. This would then be an inextendible causal curve passing through $p$ but not intersecting $\Sigma$, implying that $p$ does not belong to $D(\Sigma)$, thus contradicting the assumption.

Lemma 2. Let $R$ be a closed set such that every causal curve connecting two points in $R$ lies entirely in $R$. Let $\Sigma$ be the future boundary of $R$ defined by points $p \in R$ such that $\exists$ a timelike curve $\lambda$ passing through $p$ that does not intersect $R$ anywhere in the future. Then,

(1) $\Sigma$ is an achronal set, and

(2) $R \subseteq D(\Sigma)$.

Proof.-We first show that $\Sigma$ is an achronal set. Suppose there exist two points $p$ and $q$ in $\Sigma$ that were timelike related. Without loss of generality, assume that $p$ lies to the past of $q$. Consider an open neighborhood of $p$ denoted by $U(p)$. Consider a point $r$ such that $r \in\left\{I_{+}(p) \cap U(p)\right\} \backslash R$. By continuity, $\exists$ a timelike curve $\lambda$ connecting $r$ to $q . \lambda$ can then be extended to pass through $p$ in the past. Thus, we have found a causal curve that connects points $p$ and $q$, both of which belong to $R$, and passes through $r \notin R$. This contradicts the assumption, and hence, $\Sigma$ must be an achronal set.

Now, we can show that $R \subseteq D(\Sigma)$. Consider a point $p$ such that $p \in R \backslash \Sigma$. Then, $I_{+}(p)$ must intersect $\Sigma$. To show this, suppose it were not true and consider a future causal curve $\lambda$ from $p$ which does not intersect $\Sigma$. The boundary point of $\lambda \cap R$ then also has a timelike curve through it which does not intersect $R$ anywhere in the future, and thus should be included in the set $\Sigma$. Therefore, $I_{+}(p)$ either intersects $\Sigma$ everywhere in the interior of $\Sigma$ or intersects some portion of the boundary of $\Sigma$. In the first case, all inextendible causal curves through $p$ necessarily pass through $\Sigma$, and hence $p \in D(\Sigma)$. In the second case, extend $\Sigma$ in a spacelike manner to an open neighborhood around $\Sigma$ where a point $q$ outside $\Sigma$ is timelike related to $p$. Causal curves from $p$ to $q$ would not intersect $\Sigma$ since $q$ is spacelike related to all points on $\Sigma$. Consider the intersection of this curve with $R$. It must have a boundary point which does not belong to $\Sigma$. This point would then have inextendible timelike curves through it that do not intersect $R$ in the future. This contradicts the assumption that this point was not in $\Sigma$. This implies that the second case is impossible. Hence, we have proved that $R \subseteq D(\Sigma)$.

Theorem 1. Consider two codimension-1 spacelike subregions $\Sigma_{1}$ and $\Sigma_{2}$ that are compact. Let their domains of dependence be $D\left(\Sigma_{1}\right)=D_{1}$ and $D\left(\Sigma_{2}\right)=D_{2}$. Then $D=D_{1} \cap D_{2}$ is the domain of dependence of the future boundary of $D$ denoted by $\Sigma$.

Proof.-Consider any two points $p$ and $q$ that belong to $D$. Both $p$ and $q$ belong to $D_{1}$ and $D_{2}$. Using Lemma 1, we can conclude that all points on a causal curve joining $p$ and $q$ belong to both $D_{1}$ and $D_{2}$. Hence, any such point also belongs to $D$. Thus, $D$ satisfies the condition required for $R$ above in Lemma 2. Using Lemma 2 then tells us that $D \subseteq D(\Sigma)$.

Since $\Sigma$ is defined to be the future boundary of $D, \Sigma$ itself is necessarily contained in $D$. Now consider any $p \in D(\Sigma)$. Any causal curve $\lambda$ passing through $p$ intersects $\Sigma$ by definition. However, since $\Sigma \subseteq D$, all inextendible causal curves through $\Sigma$ necessarily intersect both $\Sigma_{1}$ and $\Sigma_{2}$. Thus, all inextendible causal curves through $p$ also pass through both $\Sigma_{1}$ and $\Sigma_{2}$. This implies $D(\Sigma) \subseteq D$.

Combining the above two results, we have shown that $D=D(\Sigma)$. Namely, the intersection of two domains of dependence is also a domain of dependence.

\section{APPENDIX B: UNIQUENESS OF THE HOLOGRAPHIC SLICE}

Consider a codimension-2, closed, achronal surface $\sigma$ in an arbitrary $(d+1)$-dimensional spacetime $M$. Suppose $\sigma$ is a convex boundary. We assume that both $M$ and $\sigma$ are sufficiently smooth so that variations in the spacetime metric $g_{\mu \nu}$ and induced metric on $\sigma$, denoted by $h_{i j}$, occur on characteristic length scales $L$ and $L_{\sigma}$, respectively.

Theorem 2. Consider a subregion $R$ of characteristic length $\delta \ll L, L_{\sigma}$ on $\sigma$. To leading order, the extremal surface anchored to $\partial R$ lives on the hypersurface generated by the vector $s=\theta_{t} t-\theta_{z} z$ normal to $\sigma$. Here, $t$ and $z$ are orthonormal timelike and spacelike vectors perpendicular to $\sigma$, and $\theta_{t}=h^{i j} K_{i j}^{t}$ and $\theta_{z}=h^{i j} K_{i j}^{z}$ where $K_{i j}^{t}$ and $K_{i j}^{z}$ are 
the extrinsic curvature tensors of $\sigma$ for $t$ and $z$, respectively. This property is independent of the shape of $R$.

Proof.-Start from a point $p \in R$ and set up Riemann normal coordinates in the local neighborhood of $p$,

$$
g_{\mu \nu}(x)=\eta_{\mu \nu}-\frac{1}{3} R_{\mu \rho \nu \sigma} x^{\rho} x^{\sigma}+O\left(x^{3}\right) .
$$

In these coordinates, we are considering a patch of size $\delta$ around the origin $p$ with $R_{\mu \rho \nu \sigma} \sim O\left(1 / L^{2}\right)$. Equivalently, we could consider a conformally rescaled metric

$$
\begin{aligned}
x^{\mu} & =\epsilon y^{\mu}, \\
d s^{2} & =\epsilon^{2} g_{\mu \nu}(\epsilon y) d y^{\mu} d y^{\nu}, \\
d \tilde{s}^{2} & =g_{\mu \nu}(\epsilon y) d y^{\mu} d y^{\nu}=\tilde{g}_{\mu \nu}(y) d y^{\mu} d y^{\nu},
\end{aligned}
$$

where $\epsilon=\delta / L \ll 1$.

In this alternate way of viewing the problem, we have a patch of size $L$ with the metric varying on a larger length scale $L / \epsilon$. In these coordinates, each derivative of the conformal metric brings out an extra power of $\epsilon$; for example,

$$
\frac{\partial^{2}}{\partial y^{\rho} \partial y^{\sigma}} \tilde{g}_{\mu \nu}=\epsilon^{2} \frac{\partial^{2}}{\partial x^{\rho} \partial x^{\sigma}} g_{\mu \nu} \sim \frac{\epsilon^{2}}{L^{2}} .
$$

The connection coefficients $\Gamma_{\rho \sigma}^{\mu}$ vanish at $p$ due to our choice of Riemann normal coordinates. This implies that for points in the neighborhood of $p$, we can Taylor expand to find

$$
\begin{aligned}
\Gamma_{\rho \sigma}^{\mu} & \sim \frac{\epsilon^{2}}{L}, \\
R_{\mu \rho \nu \sigma} & \sim \frac{\epsilon^{2}}{L^{2}} .
\end{aligned}
$$

Note that these quantities are obtained using the rescaled metric $\tilde{g}_{\mu \nu}$ in the $y^{\mu}$ coordinates.

Since there is still a remaining $S O(d, 1)$ symmetry that preserves the Riemann normal coordinate form of the metric, we can use these local Lorentz boosts and rotations to set $t$ and $z$ as the coordinates in the normal direction to $\sigma$ at $p$ while $y^{i}$ parametrize the tangential directions. This is a convenient choice to solve the extremal surface equation in a perturbation series order by order. The extremal surface equation is given by [5]

$$
\tilde{g}^{\rho \sigma}\left(\partial_{\rho} \partial_{\sigma} Y^{\mu}+\Gamma_{\lambda \eta}^{\mu} \partial_{\rho} Y^{\lambda} \partial_{\sigma} Y^{\eta}-\Gamma_{\rho \sigma}^{\lambda} \partial_{\lambda} Y^{\mu}\right)=0 .
$$

This is a set of $d+1$ equations for the embedding of the extremal surface $Y^{\mu}$, which are functions of $d-1$ independent coordinates. The equations in the tangential directions are trivially satisfied by taking the $d-1$ parameters to be $y^{i}$. This leaves only two equations in the normal directions to be solved.

From the discussion above, when restricted to the local patch of size $L$, we have

$$
\begin{aligned}
& \tilde{g}^{\mu \nu}=\eta^{\mu \nu}+O\left(\epsilon^{2}\right), \\
& \Gamma_{\rho \sigma}^{\mu}=O\left(\frac{\epsilon^{2}}{L}\right) .
\end{aligned}
$$

Assuming the extremal surface is smooth, derivatives of $Y^{\mu}$ typically bring down a power of $L_{\sigma}$. Thus,

$$
\begin{gathered}
\partial Y \sim O(1), \\
\partial^{2} Y \sim O\left(\frac{1}{L_{\sigma}}\right) .
\end{gathered}
$$

Using this, at the leading order in $\epsilon$ and $\epsilon_{\sigma}=\delta / L_{\sigma}$, the extremal surface equations simply become

$$
\delta^{i j} \partial_{i} \partial_{j} Y^{\mu}=0,
$$

where $\mu$ takes the $t$ and $z$ directions. We write these as

$$
\nabla^{2} t_{\mathrm{E}}=\nabla^{2} z_{\mathrm{E}}=0
$$

where $t_{\mathrm{E}}$ and $z_{\mathrm{E}}$ are functions of $y^{i}$.

Let $K_{i j}^{t}, K_{i j}^{z}$ denote the extrinsic curvature tensors for the $t$ and $z$ normals, respectively. Following the above scaling arguments, $K_{i j}^{t}, K_{i j}^{z} \sim \epsilon / L_{\sigma}$. Here, we have assumed that $L_{\sigma} \lesssim L$, although this is not essential for the final result. Because $t$ and $z$ are normal to the leaf, the equations for the leaf, described by $t_{\mathrm{L}}\left(y^{i}\right)$ and $z_{\mathrm{L}}\left(y^{i}\right)$, can be Taylor expanded in the neighborhood $R$ as

$$
\begin{aligned}
& t_{\mathrm{L}}\left(y^{i}\right)=-\frac{1}{2} K_{i j}^{t} y^{i} y^{j}+O\left(\frac{\epsilon^{2} y^{3}}{L_{\sigma}^{2}}\right), \\
& z_{\mathrm{L}}\left(y^{i}\right)=\frac{1}{2} K_{i j}^{z} y^{i} y^{j}+O\left(\frac{\epsilon^{2} y^{3}}{L_{\sigma}^{2}}\right),
\end{aligned}
$$

where the negative sign in the first line is due to the timelike signature of the $t$ normal. The boundary conditions for the extremal surface equation are

$$
\begin{aligned}
& t_{\mathrm{E}}(\partial R)=t_{\mathrm{L}}(\partial R), \\
& z_{\mathrm{E}}(\partial R)=z_{\mathrm{L}}(\partial R) .
\end{aligned}
$$

Now, consider $\delta t=t_{\mathrm{E}}-t_{\mathrm{L}}$ and $\delta z=z_{\mathrm{E}}-z_{\mathrm{L}}$. The extremal surface equations are then given by

$$
\nabla^{2} \delta t=-\nabla^{2} t_{\mathrm{L}}=\theta_{t}\left\{1+O\left(\epsilon_{\sigma}\right)\right\}
$$




$$
\nabla^{2} \delta z=-\nabla^{2} z_{\mathrm{L}}=-\theta_{z}\left\{1+O\left(\epsilon_{\sigma}\right)\right\}
$$

where $\theta_{t}=h^{i j} K_{i j}^{t}$ and $\theta_{z}=h^{i j} K_{i j}^{z}$. Note that $h_{i j}=\eta_{i j}$ at this order. The boundary conditions are given by

$$
\delta t(\partial R)=\delta z(\partial R)=0 .
$$

It is now clear that at leading order $\delta t / \theta_{t}$ and $-\delta z / \theta_{z}$ satisfy the same equation with the same boundary conditions. Thus,

$$
\frac{\delta t}{\delta z}=-\frac{\theta_{t}}{\theta_{z}}+O\left(\epsilon_{\sigma}\right)
$$

for all points on the extremal surface. Rewritten, the extremal surface lives on the hypersurface generated by $s=\theta_{t} t-\theta_{z} z$, orthogonal to $\sigma$.

This result is independent of the explicit shape of subregion $R$.

Theorem 2 essentially brings us to the uniqueness of the holographic slice. The new surface, $\sigma^{\prime}$, is generated by a convolution of the "deepest" points on each $\gamma(R)$. Considering balanced shapes such that the "deepest" point corresponds to $y^{i}=0, \delta t / \delta z$ has the interpretation of the slope of the evolution vector $s$ from $p$ which takes it to the new leaf $\sigma^{\prime}$. Slight imbalances in the shape would only affect the slope at subleading order in $\epsilon, \epsilon_{\sigma}$ and thus, the slope of $s$ is determined in a shape-independent manner in the limit $\epsilon, \epsilon_{\sigma} \rightarrow 0$. In order to move to $\sigma^{\prime}$, we must also specify the distance, $\delta \lambda(p)$, by which we move along $s$ at each step. If the size of $C(p)$ is homogeneous across $\sigma$, then $\delta \lambda(p)$ is independent of $p$ to leading order. Thus, the new leaf $\sigma^{\prime}$ obtained at each stage is unique up to small error terms. Following a similar procedure at each stage, e.g. by choosing random uncorrelated shapes of size $\delta^{\prime}$ (found by mapping length $\delta$ to $\sigma^{\prime}$ by $s$ ) for subregions $C^{\prime}(p)$ at each point $p$, ensures that the error terms do not add up coherently. This implies that the holographic slice is obtained by following the integral curves of the evolution vector $s$ starting from each point $p \in \sigma$, and hence is unique.

Corollary 1. Construct a holographic slice such that $C^{i}(p)$ is homogeneous and uncorrelated with $C^{j}(p), j \neq i$. Let the sizes of $C^{i}(p)$ be determined by mapping the characteristic length, $\delta$, of $C(p)$ on $\sigma$ to $\sigma^{i}$ by $s$. The continuum version (sending $\delta \rightarrow 0$ ) of all such slices are identical.

As an aside, there are certain interesting features that this analysis highlights. Consider a generic leaf of a holographic screen $\sigma$ and the future-directed orthogonal null vectors $k$ and $l$ normalized as $k \cdot l=-2$. The $t$ and $z$ vectors are then given by

$$
t=\frac{1}{2}(k+l), \quad z=\frac{1}{2}(k-l) .
$$

From the linearity of extrinsic curvature, this leads to

$$
\theta_{t}=\frac{1}{2}\left(\theta_{k}+\theta_{l}\right), \quad \theta_{z}=\frac{1}{2}\left(\theta_{k}-\theta_{l}\right) .
$$

The evolution vector $s$ and its associated expansion $\theta_{s}$ are given by

$$
s=\theta_{t} t-\theta_{z} z=\frac{1}{2}\left(\theta_{k} l+\theta_{l} k\right),
$$

and

$$
\theta_{s}=\theta_{t}^{2}-\theta_{z}^{2}=\theta_{k} \theta_{l} \leq 0
$$

respectively.

At the holographic screen, $\theta_{k}=0$. This leads to

$$
s \propto k, \quad \theta_{s}=0 .
$$

Namely, the initial evolution of the holographic slice from a nonrenormalized leaf occurs in the $k$ direction with a nonexpanding or contracting leaf area.

\section{APPENDIX C: CONVEXITY OF RENORMALIZED LEAVES}

Definition. On a spacelike slice $\Sigma$, a compact set $S$ is defined to be convex if all the codimension-1 minimal surfaces $\gamma(A)$ anchored to a codimension-2 region $A \subset S$ are such that $\forall A, \gamma(A) \subset S$.

Lemma 3. $S$ is convex if and only if $K_{\Sigma}(\partial S) \leq 0$, where $K_{\Sigma}(\partial S)$ is the trace of the extrinsic curvature of $\partial S$ embedded in $\Sigma$ for the normal pointing inward.

Proof.-This follows from the fact that if $K_{\Sigma}(\partial S) \leq 0$, $\partial S$ acts as a minimal surface barrier and hence, all the minimal surfaces must be contained within $S$. For the converse, suppose $K_{\Sigma}(\partial S)>0$ somewhere on $\partial S$; then by considering small enough subregions anchored to this portion of $\partial S$, one can explicitly construct minimal surfaces that are outside $S$.

Definition. In a spacetime $M$, a codimension- 2 compact surface $\sigma$ is called a convex boundary if on every codimension-1 spacelike slice $\Sigma$ such that $\sigma \subset \Sigma$, the closure of the interior of $\sigma$ is a convex set.

Theorem 3. $\sigma$ is a convex boundary if and only if the null expansions in the inward direction, i.e., $\theta_{k}$ and $\theta_{-l}$, are both nonpositive.

Proof.-An inward normal $n$ on a spacelike slice $\Sigma$ is given by a linear superposition of $k$ and $l$, i.e., $n=\alpha k-\beta l$ with some $\alpha, \beta \geq 0$. If $\theta_{k} \leq 0$ and $\theta_{l} \geq 0$, then $K_{\Sigma}(\sigma)=$ $\theta_{n}=\alpha \theta_{k}-\beta \theta_{l} \leq 0$ for all choices of $\alpha, \beta \geq 0$. Thus, from Lemma 3, $\sigma$ would be convex on all $\Sigma$ containing $\sigma$. For the converse, suppose $\theta_{k}>0$. One can then choose $\Sigma$ such that $K_{\Sigma}(\sigma)>0$ by taking $\beta \ll \alpha$. Thus, from Lemma $3, \sigma$ would 
not be convex on $\Sigma$, and hence $\sigma$ would not be a convex boundary. The same argument applies if $\theta_{l}<0$.

Fact. A leaf of a holographic screen is a convex boundary. The boundary of any entanglement wedge is also a convex boundary.

Theorem 4. The intersection of the interior domains of dependence of two convex boundaries $\sigma_{1}$ and $\sigma_{2}$, represented by $D_{1}$ and $D_{2}$, is the interior domain of dependence of a convex boundary $\sigma^{\prime}$.

Proof.-As shown in Appendix A, $D^{\prime}=D_{1} \cap D_{2}$ is the interior domain of dependence of some $\sigma^{\prime}$. We only need to show that $\sigma^{\prime}$ is convex. In order to show this, we can consider two slices $\Sigma_{1}$ and $\Sigma_{2}$ passing through $\sigma_{1}$ and $\sigma_{2}$ such that they are identical in the interior of $\sigma^{\prime}$ and are disjoint in the exterior of $\sigma^{\prime}$. Let us denote the slice through the interior of $\sigma^{\prime}$ as $\Sigma^{\prime}$. Now consider any codimension- 2 region $A \subset \Sigma^{\prime}$. Then, from the convexity of $\sigma_{1}$, the minimal surface $\gamma(A)$ is contained in the interior of $\sigma_{1}$ on $\Sigma_{1}$. Similarly, $\gamma(A)$ is contained in the interior of $\sigma_{2}$ on $\Sigma_{2}$. This is only possible if $\gamma(A)$ is contained in the interior of $\sigma^{\prime}$ on $\Sigma^{\prime}$. This is true for arbitrary $\Sigma^{\prime}$ and hence, by definition, $\sigma^{\prime}$ is a convex boundary. The maximin process can now be applied to $\sigma^{\prime}$.

Corollary 2. In a black hole spacetime or the case of a spacelike screen in an FRW spacetime, the coarse-graining procedure moves away from the singularity in the direction where the expansions $\theta_{k}$ and $\theta_{l}$ have opposite signs. At each step of coarse graining, $\theta_{k}$ and $\theta_{l}$ in general have opposite signs.
[1] J. Maldacena, The large $N$ limit of superconformal field theories and supergravity, Int. J. Theor. Phys. 38, 1113 (1999); The large $N$ limit of superconformal field theories and supergravity, Adv. Theor. Math. Phys. 2, 231 (1998).

[2] S. S. Gubser, I. R. Klebanov, and A. M. Polyakov, Gauge theory correlators from noncritical string theory, Phys. Lett. B 428, 105 (1998).

[3] E. Witten, Anti-de Sitter space and holography, Adv. Theor. Math. Phys. 2, 253 (1998).

[4] S. Ryu and T. Takayanagi, Holographic Derivation of Entanglement Entropy from AdS/CFT, Phys. Rev. Lett. 96, 181602 (2006).

[5] V.E. Hubeny, M. Rangamani, and T. Takayanagi, A covariant holographic entanglement entropy proposal, J. High Energy Phys. 07 (2007) 062.

[6] D. L. Jafferis, A. Lewkowycz, J. Maldacena, and S. J. Suh, Relative entropy equals bulk relative entropy, J. High Energy Phys. 06 (2016) 004.

[7] X. Dong, D. Harlow, and A. C. Wall, Reconstruction of Bulk Operators within the Entanglement Wedge in GaugeGravity Duality, Phys. Rev. Lett. 117, 021601 (2016).

[8] J. D. Bekenstein, Black holes and entropy, Phys. Rev. D 7, 2333 (1973).

[9] S. W. Hawking, Particle creation by black holes, Commun. Math. Phys. 43, 199 (1975); Erratum 46, 206 (1976).

[10] R. Bousso, A covariant entropy conjecture, J. High Energy Phys. 070 (1999) 004.

[11] G. 't Hooft, Dimensional reduction in quantum gravity, Conf. Proc. C930308, 284 (1993).

[12] L. Susskind, The world as a hologram, J. Math. Phys. (N.Y.) 36, 6377 (1995).

[13] M. Miyaji and T. Takayanagi, Surface/state correspondence as a generalized holography, Prog. Theor. Exp. Phys. 2015, 073 B03 (2015).

[14] N. Bao, S. Nezami, H. Ooguri, B. Stoica, J. Sully, and M. Walter, The holographic entropy cone, J. High Energy Phys. 09 (2015) 130.
[15] F. Sanches and S. J. Weinberg, Holographic entanglement entropy conjecture for general spacetimes, Phys. Rev. D 94, 084034 (2016).

[16] M. Rota and S. J. Weinberg, Maximin is not enough, Phys. Rev. D 97, 086013 (2018).

[17] B. Swingle, Constructing holographic spacetimes using entanglement renormalization, arXiv:1209.3304.

[18] F. Pastawski, B. Yoshida, D. Harlow, and J. Preskill, Holographic quantum error-correcting codes: toy models for the bulk/boundary correspondence, J. High Energy Phys. 06 (2015) 149.

[19] P. Hayden, S. Nezami, X.-L. Qi, N. Thomas, M. Walter, and Z. Yang, Holographic duality from random tensor networks, J. High Energy Phys. 11 (2016) 009.

[20] T. Faulkner, A. Lewkowycz, and J. Maldacena, Quantum corrections to holographic entanglement entropy, J. High Energy Phys. 11 (2013) 074.

[21] N. Engelhardt and A.C. Wall, Quantum extremal surfaces: holographic entanglement entropy beyond the classical regime, J. High Energy Phys. 01 (2015) 073.

[22] Y. Nomura, N. Salzetta, F. Sanches, and S. J. Weinberg, Toward a holographic theory for general spacetimes, Phys. Rev. D 95, 086002 (2017).

[23] Y. Nomura, P. Rath, and N. Salzetta, Classical spacetimes as amplified information in holographic quantum theories, Phys. Rev. D 97, 106025 (2018).

[24] Y. Nomura, P. Rath, and N. Salzetta, Spacetime from unentanglement, Phys. Rev. D 97, 106010 (2018).

[25] R. Bousso, Holography in general space-times, J. High Energy Phys. 06 (1999) 028.

[26] D. N. Page, Average Entropy of a Subsystem, Phys. Rev. Lett. 71, 1291 (1993).

[27] J. Haegeman, T. J. Osborne, H. Verschelde, and F. Verstraete, Entanglement Renormalization for Quantum Fields in Real Space, Phys. Rev. Lett. 110, 100402 (2013). 
[28] R. Bousso and N. Engelhardt, New Area Law in General Relativity, Phys. Rev. Lett. 115, 081301 (2015).

[29] R. Bousso and N. Engelhardt, Generalized second law for cosmology, Phys. Rev. D 93, 024025 (2016).

[30] A. Hamilton, D. N. Kabat, G. Lifschytz, and D. A. Lowe, Holographic representation of local bulk operators, Phys. Rev. D 74, 066009 (2006).

[31] A. Hamilton, D. N. Kabat, G. Lifschytz, and D. A. Lowe, Local bulk operators in AdS/CFT: a holographic description of the black hole interior, Phys. Rev. D 75, 106001 (2007).

[32] I. Heemskerk, D. Marolf, J. Polchinski, and J. Sully, Bulk and transhorizon measurements in AdS/CFT, J. High Energy Phys. 10 (2012) 165.

[33] R. Bousso, S. Leichenauer, and V. Rosenhaus, Light-sheets and AdS/CFT, Phys. Rev. D 86, 046009 (2012).

[34] B. Czech, J. L. Karczmarek, F. Nogueira, and M. Van Raamsdonk, The gravity dual of a density matrix, Classical Quantum Gravity 29, 155009 (2012).

[35] A. C. Wall, Maximin surfaces, and the strong subadditivity of the covariant holographic entanglement entropy, Classical Quantum Gravity 31, 225007 (2014).

[36] M. Headrick, V.E. Hubeny, A. Lawrence, and M. Rangamani, Causality \& holographic entanglement entropy, J. High Energy Phys. 12 (2014) 162.

[37] E. T. Akhmedov, A remark on the AdS/CFT correspondence and the renormalization group flow, Phys. Lett. B 442, 152 (1998).

[38] E. Álvarez and C. Gómez, Geometric holography, the renormalization group and the c-theorem, Nucl. Phys. B541, 441 (1999).

[39] V. Balasubramanian and P. Kraus, Space-Time and the Holographic Renormalization Group, Phys. Rev. Lett. 83, 3605 (1999).

[40] K. Skenderis and P. K. Townsend, Gravitational stability and renormalization group flow, Phys. Lett. B 468, 46 (1999).

[41] J. de Boer, E. Verlinde, and H. Verlinde, On the holographic renormalization group, J. High Energy Phys. 08 (2000) 003.

[42] L. Andersson and J. Metzger, The area of horizons and the trapped region, Commun. Math. Phys. 290, 941 (2009).

[43] M. Mars, Stability of marginally outer trapped surfaces and geometric inequalities, Fund. Theor. Phys. 177, 191 (2014).

[44] F. Sanches and S. J. Weinberg, Refinement of the BoussoEngelhardt area law, Phys. Rev. D 94, 021502 (2016).

[45] F. Sanches and S. J. Weinberg, Boundary dual of bulk local operators, Phys. Rev. D 96, 026004 (2017).

[46] R. Espíndola, A. Guijosa, and J. F. Pedraza, Entanglement wedge reconstruction and entanglement of purification, arXiv: 1804.05855.

[47] N. Bao and I. F. Halpern, Holographic inequalities and entanglement of purification, J. High Energy Phys. 03 (2018) 006.

[48] N. Bao (private communication).

[49] V. Balasubramanian, B. D. Chowdhury, B. Czech, and J. de Boer, Entwinement and the emergence of spacetime, J. High Energy Phys. 01 (2015) 048.
[50] N. Engelhardt and A. C. Wall, Extremal surface barriers, J. High Energy Phys. 03 (2014) 068.

[51] B. Freivogel, R. A. Jefferson, L. Kabir, B. Mosk, and I.-S. Yang, Casting shadows on holographic reconstruction, Phys. Rev. D 91, 086013 (2015).

[52] V.E. Hubeny and H. Maxfield, Holographic probes of collapsing black holes, J. High Energy Phys. 03 (2014) 097.

[53] L. Susskind, L. Thorlacius, and J. Uglum, The stretched horizon and black hole complementarity, Phys. Rev. D 48, 3743 (1993).

[54] Y. Nomura and N. Salzetta, Butterfly velocities for holographic theories of general spacetimes, J. High Energy Phys. 10 (2017) 187.

[55] C. Fefferman and C. R. Graham, Conformal invariants, in The Mathematical Heritage of Élie Cartan (Société Mathématique de France, Lyon, 1984).

[56] H. Bondi, M. G. J. van der Burg, and A. W. K. Metzner, Gravitational waves in general relativity VII. Waves from axisymmetric isolated systems, Proc. R. Soc. A 269, 21 (1962).

[57] R. K. Sachs, Gravitational waves in general relativity VIII. Waves in asymptotically flat space-times, Proc. R. Soc. A 270, 103 (1962).

[58] M. Nozaki, S. Ryu, and T. Takayanagi, Holographic geometry of entanglement renormalization in quantum field theories, J. High Energy Phys. 10 (2012) 193.

[59] T. Takayanagi and K. Umemoto, Holographic entanglement of purification, Nat. Phys. 14, 573 (2018).

[60] P. Nguyen, T. Devakul, M. G. Halbasch, M. P. Zaletel, and B. Swingle, Entanglement of purification: from spin chains to holography, J. High Energy Phys. 01 (2018) 098.

[61] T. Faulkner and A. Lewkowycz, Bulk locality from modular flow, J. High Energy Phys. 07 (2017) 151.

[62] G. Vidal, Entanglement Renormalization, Phys. Rev. Lett. 99, 220405 (2007).

[63] Y. Nomura and J. Varela, A note on (no) firewalls: the entropy argument, J. High Energy Phys. 07 (2013) 124.

[64] N. Bao, S. M. Carroll, A. Chatwin-Davies, J. Pollack, and G. N. Remmen, Branches of the black hole wave function need not contain firewalls, arXiv:1712.04955 [Phys. Rev. D (to be published)].

[65] Y. Nomura, F. Sanches, and S. J. Weinberg, Black Hole Interior in Quantum ravity, Phys. Rev. Lett. 114, 201301 (2015).

[66] Y. Nomura and N. Salzetta, Why firewalls need not exist, Phys. Lett. B 761, 62 (2016).

[67] L. Susskind and E. Witten, The holographic bound in antide Sitter space, arXiv:hep-th/9805114.

[68] D. Harlow, The Ryu-Takayanagi formula from quantum error correction, Commun. Math. Phys. 354, 865 (2017).

[69] A. Almheiri, X. Dong, and B. Swingle, Linearity of holographic entanglement entropy, J. High Energy Phys. 02 (2017) 074. 